\title{
PHOTONIC BLOCH WAVES AND PHOTONIC BAND GAPS
}

\author{
Philip St.J. Russell, Timothy A. Birks and F. Dominic Lloyd-Lucas \\ Optoelectronics Research Centre, \\ University of Southampton, \\ Hants SO9 5NH, United Kingdom
}

\section{INTRODUCTION}

Photonic band gap materials are dielectrics with a synthetic, three dimensional, multiply periodic microstructure (lattice constant of order the optical wavelength) whose distinguishing feature is a very large modulation depth of refractive index. When appropriately designed, these "photonic crystals" exhibit ranges of optical frequency where light cannot exist - the photonic band gaps ${ }^{7}$. The current interest in these materials ${ }^{1-22}$ has led us to re-appraise propagation in structures that, while not exhibiting a complete photonic band gap (PBG), nevertheless display anomalous and intriguing propagation effects in the vicinity of their Bragg conditions ${ }^{54-58}$. In most cases, around each Bragg condition appear incomplete momentum and energy gaps (i.e., ranges of, respectively, wavevector and frequency where propagation is forbidden) with widths that are given approximately by the product of the index difference with, respectively, the vacuum wavevector and $h$ times the optical frequency. With the exception of the multi-layer dielectric stack, most conventional electromagnetic gratings, such as those encountered in holography ${ }^{27}$, waveguides ${ }^{45}$, distributed feedback lasers ${ }^{35,37,38}$, acousto-optic ${ }^{47}$ and $x$-ray ${ }^{61}$ diffraction, consist of weak periodic perturbations about a mean refractive index. In these gratings, while strong spatial and temporal dispersion are present around each Bragg condition, the ranges of angles and frequencies over which this occurs are very narrow; and although PBG's do appear, they are incomplete and mostly very weak.

It was in this context that Yablonovitch posed the question: By analogy with electronic band gaps, would a full photonic band gap appear in a multiply periodic threedimensional structure if the refractive index were very strongly modulated? As we now know $^{7}$, state-of-the-art optics was unable to answer the question immediately, because although large modulation depths were well known and accepted in the solid state physics of electrons, where the lattice potential is often both very deeply modulated and highly non-sinusoidal ${ }^{23}$, they were encountered in optics only in the form of singly periodic multilayer stacks. Commonly-used and well-understood perturbation approaches ${ }^{27}$, in which the field in a singly periodic structure is Fourier decomposed into a finite set of plane waves coupled together by the lattice, were not immediately applicable to the full vector case of multiply periodic three-dimensional structures. The high modulation depth of index in PBG structures also means that even the concept of average index is of limited usefulness; as we shall see, this is because the expectation value of the refractive index can deviate 
hugely from its mean value via re-distribution of the photon probability function (or optical intensity) into regions of higher or lower dielectric constant ${ }^{5}$. Indeed, this redistribution, which depends on $\omega$, lies behind all the complex and curious types of behaviour encountered in photonic band gap materials.

The field of photonic band structure is a hybrid, drawing on the resources of two major disciplines: optics and electronic band theory. Electronic band theory is rich in concepts $^{23-25}$ (such as density of states and effective mass) not widely used in optics, where the emphasis is more on wave propagation ${ }^{43-50}$. On the other hand, conventional optics provides a range of ideas (such as rays, diffraction, refraction and interference) that are essential if photonic band gap materials are to be used in real systems, where propagation in the vicinity of the band gap must be well understood. For example, the effective mass method $^{23,25}$ turns out to be useful for treating the propagation of Bloch rays in photonic band gap structures with slowly-varying non-uniformities, whereas generalised versions of refraction and dispersion ${ }^{57}$ are needed to treat wave behaviour at sharp interfaces between different periodic media.

The theme of this chapter is thus the development of an approach to propagation in periodic optical media that uses Bloch waves instead of plane waves; and the goal is to provide the basis for a full "quantum photo-dynamical" (QPD) description of light in PBG structures, where the density of photonic states is controlled by the presence of a dielectric "crystal" lattice.

The simplicity of the multi-layer dielectric stack, and the availability of exact analytical solutions for the Bloch waves it supports ${ }^{28,29}$, make it an ideal vehicle for illustrating the physics of photonic band gaps. A major aim of this chapter is to reinterpret the behaviour of the multi-layer stack within the framework of photonic band structure, making use of versatile intuitive graphical tools such as wavevector $(k-k)$, Brillouin $(\omega-k)$ and band-edge diagrams. The physical origins of energy and momentum band gaps are discussed, together with concepts like effective mass, group and phase velocity, and density of states of "valence" and "conduction" photons. The link between photonic Bloch waves and traditional plane wave optics is also explored, the effects of optical nonlinearities and gain briefly touched upon, and a number of unusual structures and devices described.

Note that there is a list of mathematical symbols in Appendix A1.

\subsection{List of Chapter Headings}

1. Introduction

1.1 List of chapter headings

1.2 Essential background material

1.2.1 Three regimes of behaviour

1.2.2 Hard and soft reflections

1.2.3 Brewster's angle

1.2.4 Two parallel interfaces

1.2.5 Anti-reflection condition

1.2.6 Micro-cavity resonators in two and three dimensions

2. Photons and Electrons

2.1 Wave equation for electrons and photons

2.2 Effective mass of electrons and photons in uniform isotropic media

3. Analysis of Singly Periodic Structures

3.1 Translation matrix formalism

3.2 Normalised parameters

4. Singly Periodic Structures at Normal Incidence $(\beta=0)$

4.1 Brillouin diagrams

4.2 Group velocity and density of states

4.3 Expectation values of potential: Electrons and photons

4.4 Origins of band gaps 
5. The Wavevector Diagram

6. Singly Periodic Structures at Oblique Incidence $(\beta>0)$

$6.1 \quad$ Band-edge diagrams

6.2 Photonic band gaps and windows

6.3 Points of zero band gap width

6.4 Brillouin diagrams for finite $\beta$

7. Two-Dimensional Propagation

7.1 Effective mass method for non-uniform periodic structures

7.2 Illustrations from experiments on planar waveguides

8. Extensions to Multiply Periodic Structures

9. Miscellaneous Topics

9.1 Localised modes, defects and surface-guided states

9.2 Effects of optical gain: Lasers

9.3 Effects of optical nonlinearity: Gap solitons

9.4 Applications of photonic band gap materials

10. In Conclusion

11. Acknowledgements

12. References and Reading Guide

Appendices

A1. Main Mathematical Symbols and Their Meanings

A2. Translation Matrix Elements

A3. Bessel Function Solutions

A3.1 Nearly-free photons: Green's function solution

A3.2 Tight binding

\subsection{Essential Background Material}

A range of elementary results can be derived by considering the reflection and refraction of a plane wave incident on the interface between two isotropic dielectric media $^{43,44}$. Since a good intuitive understanding of the physics of this phenomenon is vital in the ensuing treatment of photonic band structure, it will now be briefly discussed; for a more detailed account, the reader is referred to any standard optics textbook such as Born and Wolf ${ }^{44}$. The electric field of a linearly polarised electromagnetic wave in medium $j$ may be written in the form:

$$
\mathbf{E}_{j}=\mathbf{E}_{j o} \exp \left[-\mathrm{j}\left(\beta z \pm p_{j} y\right)+\mathbf{j} \omega t\right]
$$

where

$$
p_{j}=\sqrt{k^{2} n_{j}^{2}-\beta^{2}}
$$

is the wavevector component - normal to the interface - of the field within each medium, $k=\omega / c$ is the vacuum wavevector at optical frequency $\omega / 2 \pi, n_{j}$ the refractive index and $\beta$ the wavevector along the interface, whose normal points in the $y$ direction. Convention defines a transverse electric (TE, or $s$-polarised) wave as one whose electric field is parallel to the interface, and a transverse magnetic (TM, or $p$-polarised) wave as one whose electric field points in the $(y, z)$ plane. Equation $(2)$ is a consequence of the requirement that the wavevector must have a magnitude equal to $k n_{j}$; this gives rise to the very useful wavevector diagram, which is the locus of allowed wavevectors at fixed optical frequency. For isotropic media, it is a circle of radius $k n_{j}$, and for two different media, two concentric circles appear (see Figure $1 ; n_{1}>n_{2}$ is assumed throughout this chapter). In any collision of a plane wave with a plane parallel interface, the momentum along the interface is conserved; this is another way of saying that the effective wavelength along the interface of all the participating waves must be identical, i.e., that they must be phasematched. A line drawn normal to the interface, displaced from the origin by $\beta$, intersects 

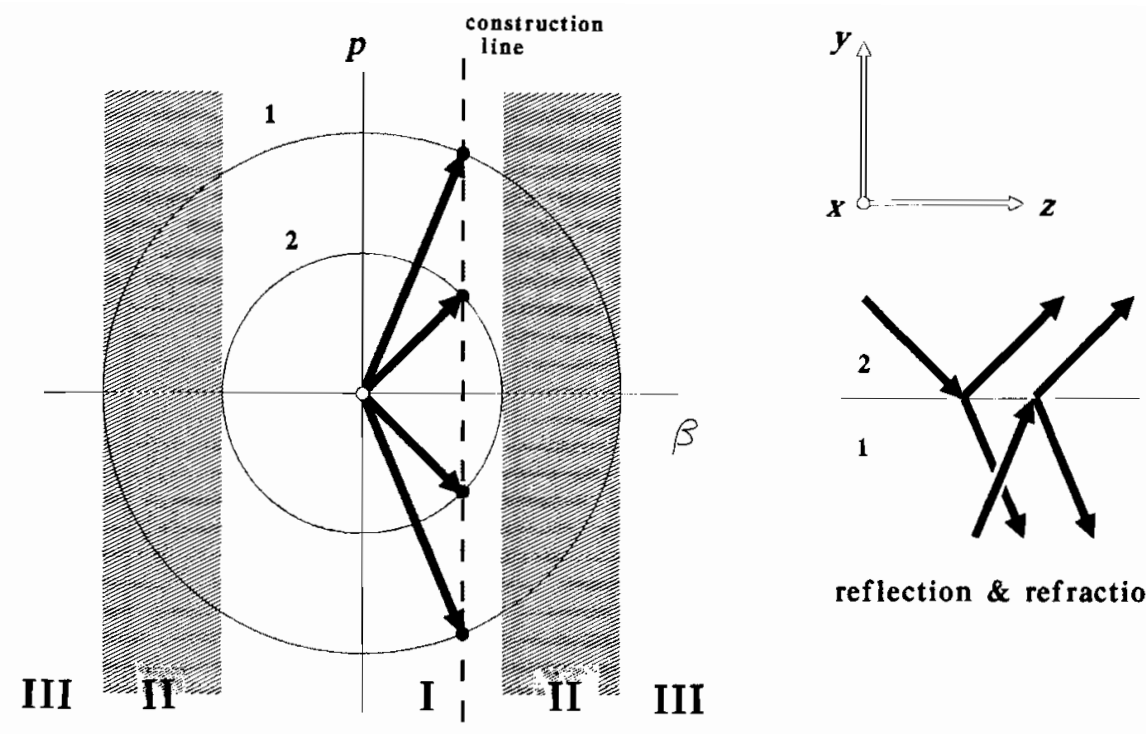

reflection \& refraction

Figure 1. Interface between two media of refractive indices $n_{1}>n_{2}$, together with the related wavevector diagram. Three regions (I, II and III) exist, corresponding to reflection/refraction, total internal reflection, and cut-off. A simple construction yields all the real-valued wavevectors that may exist at fixed wavevector $\beta$ along the interface (illustrated for region I). The ray directions of the plane waves in real space are sketched on the right hand side.

the circles at a number of points, giving the complete set of wavevectors satisfying this interface condition.

1.2.1 Three regimes of behaviour. Note that three distinct regions of behaviour exist. In the first (region I: $0<\beta<k n_{2}$ ) both refraction and reflection occur: light propagates in both media. In the second (region II: $k n_{2}<\beta<k n_{1}$ ), total internal reflection occurs: light propagates only in the high index medium. In the third (region III: $k n_{1}<\beta$ ) the light is evanescent in both media: it is cut-off from propagation. On the boundary between regions I and II the angle between the wavevector on the high index circle and the vertical axis is the critical angle.

1.2.2 Hard and soft reflections. A very important physical quantity is the phase change upon reflection. For incidence from the low index side (medium 2), a phase retardation of $\pi$ occurs throughout region I (in regions II and III the light is cut-off); the associated reflection is sometimes described as hard. For incidence from the high index side (medium 1), the phase change is zero in region I, and varies smoothly from zero to a retardation of $\pi$ across region II; in region I the reflection is sometimes described as soft.

1.2.3 Brewster's angle. The magnitude of the reflection can go to zero in the case of TM polarisation; this occurs in region I at Brewster's angle, when the refracted and reflected wavevectors are at right angles. Under these circumstances, the electric dipoles of the refracted and reflected waves are orthogonal, resulting in zero reflected power. It is easy to show (see Figure 2) that the Brewster condition is satisfied when: 

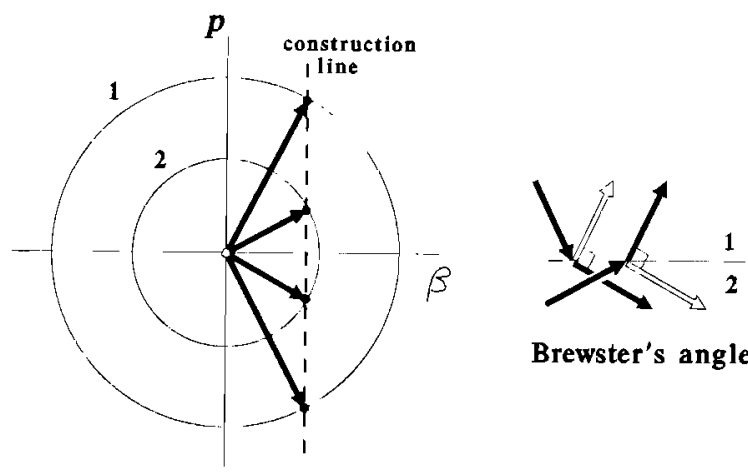

Brewster's angle

Figure 2. In region I, when the reflected and refracted rays are orthogonal, the dipoles excited by the refracted wave are unable to radiate into a reflected wave, resulting in zero reflection. This occurs at Brewster's angle.

$$
\beta=\frac{k n_{1} n_{2}}{\sqrt{n_{1}{ }^{2}+n_{2}{ }^{2}}} ;
$$

this condition applies irrespective of whether the light is incident from the low or the high index medium. No Brewster phenomenon occurs in regions II and III. Note that as the index difference tends to zero, the Brewster angle occurs at close to $45^{\circ}$ as should be expected.

1.2.4 Two parallel interfaces. When a second parallel interface is introduced, a number of new phenomena occur, the most important for our purposes being a) guided modes, b) anti-reflections and c) tunnelling across low index layers. Guided modes can form in region II for a high index layer bounded by low index media (Figure 3a). They occur when the round-trip phase change across the layer, including the phase retardation upon total internal reflection at the interfaces, equals a multiple of $2 \pi$. They may be viewed as isolated micro-resonances of the layer, and in this respect bear some resemblance to electrons trapped in atomic orbitals. Their dispersion relation takes the form (an excellent derivation is available in Kogelnik's chapter in reference 45):

$$
p_{1} h_{1}-2 \arctan \left[\frac{\xi_{2} \sqrt{-p_{2}^{2}}}{\xi_{1} p_{1}}\right]=m \pi
$$

where $h_{1}$ is the layer thickness, $m$ the mode order and the $\xi_{j}$ contain the polarization dependence:

$$
\xi_{j}=1 \text { (TE) or } 1 / n_{j}^{2} \text { (TM). }
$$

Retaining these factors allows both the TE and TM cases to be covered in a single analysis. 
Note that, unlike in the electron case, light cannot be trapped at $\beta=0$ in a high index layer since total internal reflection is impossible in dielectrics at normal incidence. Tunnelling through a thin low index layer sandwiched between two high index media can occur in region II (Figure 3b); this is important if two or more identical high index layers are brought in close proximity. Resonant tunnelling of light between waveguide microresonances can then occur, as will be discussed in more detail in section 6 .

1.2.5 Anti-reflection condition. A single layer will exhibit zero reflection when the round-trip phase change in region $I$ is an odd multiple of $\pi$, i.e., when the waves reflected from each interface interfere destructively; since the optical round-trip across any

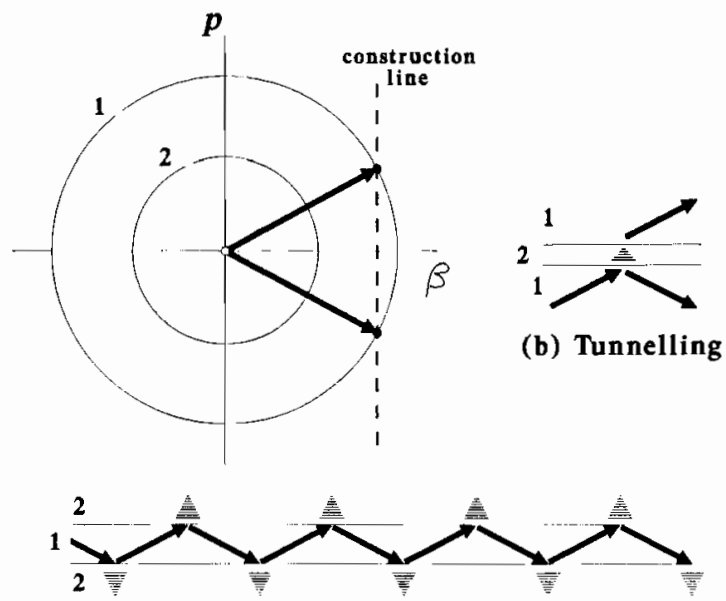

(a) Resonant guided mode

Figure 3. In region II: (a) a single layer of high index, sandwiched between two media of lower index, will support guided modes in its "potential well" for discrete values of $\beta$; (b) on the other hand, if the indices are the opposite way around, tunnelling of photons through the low index "potential barrier" is possible.

layer (of high or low index) always contains one hard and one soft reflection, the antireflection condition is ${ }^{44}$ :

$$
2 p_{i} h_{i}-\pi=(2 m-1) \pi \Rightarrow p_{i} h_{i}=m \pi
$$

which shows that, at normal incidence, the layer must be a multiple of half a wavelength thick. In the same way it may be shown that layers whose round-trip phase change is an even multiple of $\pi$ reflect strongly.

1.2.6 Micro-cavity resonators in two and three dimensions. A single island of high index material imbedded in a low index background (Figure 4a) will support a large 

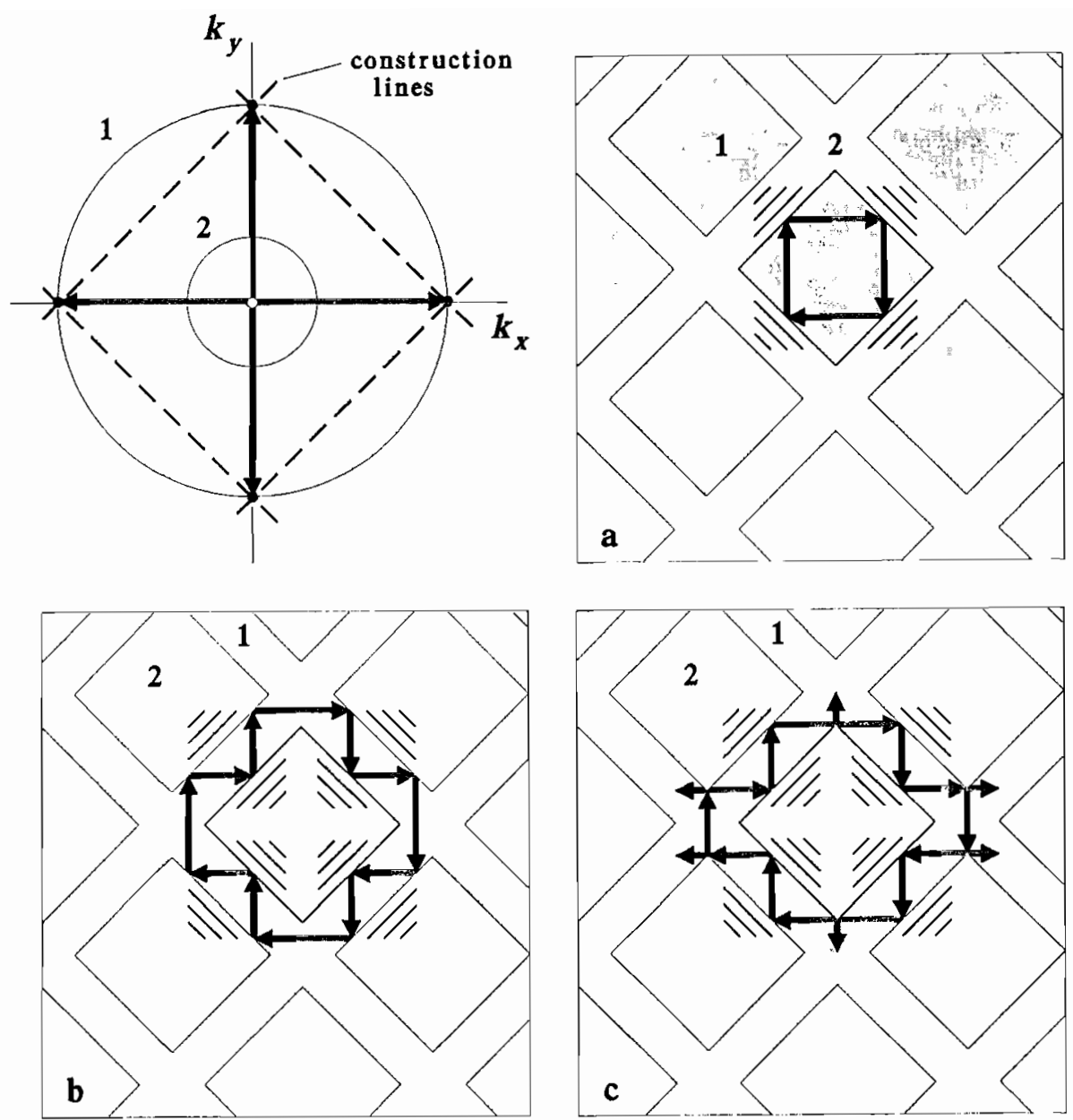

Figure 4. Cubic arrays of square unit cells (GaAs/air, critical angle $16.6^{\circ}$ ), together with their wavevector diagram for horizontal and vertical rays. High index islands (a): Certain closed ray paths (of which one is illustrated) can be found for which the round-trip phase change is a multiple of $2 \pi$; these represent leaky isolated resonances (see text). An array of identical micro-resonators will be able to "talk" to one another through their evanescent external fields, creating the conditions for resonant tunnelling. Low index islands (b)\&(c): If the high and low index regions are reversed, bound ray paths are much more difficult to find (particularly for rays at arbitrary angles to the horizontal), and any resulting micro-resonances will have very low $Q$ factors.

number of bound rays, i.e., rays which are trapped by total internal reflection (TIR). If closed paths can be found for which (at a given frequency) the round-trip phase change is a multiple of $2 \pi$, then localised resonances will appear. The Q-factor of these resonances will depend on the rate of leakage of energy into the low index surrounding medium. This in turn is governed by the width of the angular plane wave spectrum of the beamlet surrounding the trapped ray. For a very small resonator, this is large, and a significant number of plane waves will approach the boundaries outside the range of TIR. A square resonator of GaAs surrounded by air is depicted in Figure 4a, together with its wavevector diagram. The larger the index contrast, the smaller the inner circle, and the larger the number of rays within the high index material that are confined (in other words, the wider the range of total internal reflection). A perfectly confining resonator would be one for which there is no inner circle, resulting in a TIR range of $360^{\circ}$. This occurs when the 
resonator is surrounded by a perfect metal (dielectric constant negative).

The impossibility of realising perfect resonators using dielectrics means that the conditions for resonant tunnelling, across a periodic array of identical high index islands, are only ever approximately attained. Even so, under the correct excitation conditions (frequency and wavevector), the light will tend to be pulled into the high index regions, resulting in a local narrowing of the band gap and a radical shifting of its mean position (caused by a sudden change in perceived refractive index). The higher the index contrast, the more dramatic is this effect. It is therefore desirable, for attainment of a good band gap, to avoid the appearance of micro-resonances as far as possible - one does not wish to prevent the natural tendency of the light to redistribute itself into high and low index regions on (respectively) the low and high frequency sides of the band gap (see section 4.4). As Yablonovitch has demonstrated, micro-resonances may be avoided by building structures in which low index islands are imbedded in a high index medium (Figure 4b\&c). This results in a high degree of interconnection between the high index regions, effectively reducing the probability of finding strong micro-resonances - and even if they do exist, they will have very low $Q$ factors owing to the high probability of finding unbound rays.

\section{PHOTONS AND ELECTRONS}

In this section, comparisons between electrons and photons are drawn in a number of contexts relevant to the chapter's aims, with special reference to the wave equation and the concept of effective mass in uniform isotropic media.

\subsection{Wave Equation for Electrons and Photons}

Schrödinger's equation, in time-independent form, for electrons of total energy $H_{e}$ in an arbitrary potential $U$ is:

$$
\left[-\frac{\hbar^{2}}{2 m_{e}} \nabla^{2}+U(\mathbf{r})\right] \psi=H_{e} \psi
$$

where $m_{e}$ is the electron rest mass. In the absence of free charges in non-magnetic materials, Maxwell's equations (SI units) take the form ${ }^{26}$.

$$
\begin{gathered}
\nabla \times \mathbf{E}=-\mu_{o} \frac{\partial \mathbf{H}}{\partial t} \\
\nabla \times \mathbf{H}=\epsilon_{o} \varepsilon_{r} \frac{\partial \mathbf{E}}{\partial t} \\
\nabla \cdot\left(\epsilon_{o} \epsilon_{r} \mathbf{E}\right)=0, \quad \nabla \cdot\left(\mu_{o} \mathbf{H}\right)=0
\end{gathered}
$$

where the electric and magnetic fields are $\mathbf{E}$ and $\mathbf{H}$, the free space magnetic permeability and dielectric susceptibility $\mu_{o}$ and $\epsilon_{o}$ and the relative dielectric constant is $\epsilon_{r}$. Noting from (1) that $\partial / \partial t \equiv j \omega$, the following time-independent wave equation for the electric field $\mathbf{E}$ is obtained after some straightforward manipulation:

$$
\left[-\nabla^{2}-k^{2}\left\{\epsilon_{r}(\mathbf{r})-1\right\}\right] \mathbf{E}-\nabla\left[\left\{\nabla \ln \epsilon_{r}(\mathbf{r})\right\} \cdot \mathbf{E}\right]=k^{2} \mathbf{E} .
$$

The terms in (9) may be regrouped in a number of equivalent ways; we have chosen to separate out the dielectric susceptibility $\left(\epsilon_{r}-1\right)$ and interpret it as being related to potential 
energy. A dimensionally accurate comparison may be obtained by using the arbitrarily defined mass:

$$
m_{0}=\hbar \omega / 2 c^{2}
$$

which yields:

$$
\left[-\frac{\hbar^{2}}{2 m_{0}} \nabla^{2}+U_{o}(\mathbf{r})\right] \mathbf{E}-\frac{\hbar^{2}}{2 m_{o}} \nabla\left[\left\{\nabla \ln \epsilon_{r}(\mathbf{r})\right] \cdot \mathbf{E}\right]=H_{o} \mathbf{E}
$$

where

$$
U_{o}(r)=-\hbar \omega\left[\epsilon_{r}(\mathbf{r})-1\right], \quad H_{o}=\hbar \omega
$$

are the potential and total energy terms respectively; the subscript "o" means optical. The definition of "potential energy" yields $U_{o}=0$ in vacuum, which seems reasonable; note also that $U_{o}$ is negative in a dielectric material. This implies that, unlike electrons which are free only if $H_{e}$ exceeds $U$, photons are free in a dielectric at all energies since both $U_{o}$ and $H_{o}$ scale with optical frequency. Photons can of course be trapped (also at all energies) in a cavity filled with dielectric and surrounded by a perfect metal for which $\epsilon_{r}<-1$; however, this type of cavity has little practical utility since metals exhibit high dissipative losses at optical frequencies. The other obvious difference between electrons and photons lies in the vector nature of the optical fields, which produces an extra non-zero term if the susceptibility has a non-zero gradient in the direction of the electric field - something which will normally happen in photonic band gap materials. Its effect is to couple together the cartesian field components, making the calculation of band structure for photons significantly more difficult than for electrons. If it did not exist, each field component would satisfy a scalar Helmholtz equation independently, greatly reducing the complexity of the problem; the electron and photon wave equations would then be formally identical at constant optical frequency.

\subsection{Effective Mass of Electrons and Photons in Uniform Isotropic Media}

It is known that the electronic effective mass is profoundly affected by the presence of a periodic potential ${ }^{23}$; in order to understand the implications of this for photons in PBG materials it is essential first to explore and understand the comparison in the simpler nonperiodic case of a uniform isotropic medium. The matrix elements of the reciprocal effective mass tensor are given by:

$$
\frac{1}{m_{i j}^{*}}=\frac{1}{\hbar^{2}} \frac{\partial^{2} H}{\partial k_{i} \partial k_{j}}=\frac{1}{\hbar} \frac{\partial^{2} \omega}{\partial k_{i} \partial k_{j}} .
$$

Evaluation of the effective mass tensor thus requires an exact knowledge of the dispersion relations, which take the simple forms:

$$
H_{e}=\frac{\hbar^{2}\left(k_{x}^{2}+k_{y}^{2}+k_{z}^{2}\right)}{2 m_{e}}+U
$$

for electrons and 
Table I. Electrons and Photons in Isotropic Uniform Media

\begin{tabular}{|c|c|c|}
\hline compared item & electrons & photons \\
\hline particle type & fermion & boson \\
\hline occupancy per state & two (opposite spins) & $\begin{array}{l}\text { limited only by material } \\
\text { breakdown or nonlinearity }\end{array}$ \\
\hline fields & scalar & vector \\
\hline potential energy & $\begin{array}{l}U-\text { constant (effective } \\
\text { one-electron potential) }\end{array}$ & $\begin{array}{l}U_{o}=-\hbar \omega\left(\epsilon_{r}-1\right) \\
\text { (our chosen definition) }\end{array}$ \\
\hline total energy & $H_{e}$ variable & $H_{o}=\hbar \omega$ \\
\hline dispersion relation & $\mathbf{k}^{2}=2 m_{e}\left(H_{e}-U\right) / \hbar^{2}$ & $\mathbf{k}^{2}=(\omega n / c)^{2}$ \\
\hline effective mass & rest mass $m_{e}$ (scalar) & $\begin{array}{l}\infty \text { (direction of travel) } \\
2 m_{o} n \text { (for deflection) }\end{array}$ \\
\hline $\begin{array}{l}\text { evanescence at normal } \\
\text { incidence (i.e., } \beta=0 \text { )? }\end{array}$ & yes (if $H_{\varepsilon}$ small enough) & impossible in dielectrics \\
\hline polarisation effects & none & Brewster's angle \\
\hline critical angle $\theta_{c}$ & $\begin{array}{l}\arcsin \sqrt{ }\left[\left(H_{e}-U_{l}\right) /\left(H_{e}-U_{2}\right)\right] \\
\text { depends on } H_{e}\end{array}$ & $\begin{array}{l}\arcsin \left(n_{2} / n_{1}\right) \\
\text { independent of } H_{o}\end{array}$ \\
\hline in potential well at $\beta=0$ & $\begin{array}{l}\text { tight binding to free } \\
\text { electrons as } H_{e} \text { rises }\end{array}$ & $\begin{array}{l}\text { no binding possible in } \\
\text { dielectrics }\end{array}$ \\
\hline in potential well at $\beta>0$ & $\begin{array}{l}\text { tight binding to free } \\
\text { electrons as } H_{e} \text { rises }\end{array}$ & $\begin{array}{l}\text { discrete tightly bound } \\
\text { modes beyond } \theta_{c}\end{array}$ \\
\hline
\end{tabular}

$$
H_{0}=\frac{\hbar c}{n} \sqrt{k_{x}^{2}+k_{y}^{2}}+\bar{k}_{z}^{2}
$$

for photons, where $n$ is the refractive index. Deriving the reciprocal effective mass tensor is straightforward, and after diagonalisation leads to the principal forms:

$$
\left[1 / \mathbf{m}_{\mathrm{e}}^{*}\right]=\left(1 / m_{e}\right) \mathbf{I}
$$

for electrons (where $\mathbf{I}$ is the identity matrix) and

$$
\left[1 / \mathbf{m}_{0}^{*}\right]=\frac{1}{2 m_{o} n}\left[\begin{array}{lll}
1 & 0 & 0 \\
0 & 1 & 0 \\
0 & 0 & 0
\end{array}\right]
$$

for photons travelling in the $z$-direction (the quantity $m_{o}$ was defined in (10)). The electron effective mass is, as expected, a scalar quantity equal to the rest mass; electrons can be accelerated equally easily in any direction, including the direction in which they are moving. For photons, however, the situation is considerably more complicated. It turns out that the effective mass is infinite in the direction of propagation, and equal to $2 m_{D} n$ in the two directions orthogonal to this; the implication is that photons can be deflected but not accelerated. The profound differences between photons and electrons are all the more 
extraordinary when one considers that the only difference is a square root in the dispersion relation! The comparison between electrons and phoions in uniform isotropic media is summarised in Table $I$.

\section{ANALYSIS OF SINGLY PERIODIC STRUCTURES}

We present now a full two-dimensional treatment (using the translation matrix method $^{29}$ ) of singly periodic multi-layer structures, with an emphasis on re-interpretation (using the concepts of photonic band structure) and building up intuition. Periodic structuring of a medium can affect the density of states profoundly, either reducing it to zero within the photonic band gaps, or increasing it and creating new states with unusual properties in the vicinity of the photonic band gaps. A full two-dimensional treatment permits the building up of a canon of basic concepts useful for understanding these effects (and others) in more complicated multiply-periodic two and three dimensional structures. It provides an excellent introduction to the subject, illustrating simply and precisely a number of key physical concepts such as the tightly bound and nearly-free photon approximations, the Brillouin diagram, the constant energy (wavevector) diagram, the factors governing the appearance and disappearance of the band gaps, and the role of effective mass. In the alternative language of optics, photonic Bloch waves are the normal optical modes of a periodic structure, in the same sense that plane waves are the normal modes of free space.

\subsection{Translation Matrix Formalism}

This section contains a detailed mathematical account of the translation matrix method, and may be omitted at a first reading; it provides a source of the mathematical tools needed if the reader wishes at some later date to treat a specific case.

We consider a periodic medium composed of alternating planar layers with constant refractive indices $n_{1}$ and $n_{2}\left(n_{1}>n_{2}\right)$ and widths $h_{1}$ and $h_{2}$. The stack period $\Lambda$ is the sum of $h_{1}$ and $h_{2}$. The same geometry as in section 1.2 is used: cartesian axes are oriented with $y$ normal to the layer boundaries and $z$ along the layers (Figure 5); there is no field variation with $x$. As already pointed out, one key feature of the planar geometry is the separation of the electric field into TM and TE polarization states, with $E_{x}=H_{y}=H_{z}=$ 0 and $H_{x}=E_{y}=E_{z}=0$ respectively. In each case, all field components can be expressed in terms of the surviving $x$ component (denoted here by $f$ ), which itself satisfies a Helmholtz equation in each layer $(j=1,2)$ :

$$
\frac{d^{2} f_{j}}{d y^{2}}+\left(k^{2} n_{j}^{2}-\beta^{2}\right) f_{j}=0
$$

(where $\beta$ is the propagation constant in the $z$ direction) with scalar boundary conditions at the interfaces:

$$
f_{1}=f_{2}, \quad \xi_{1} \frac{d f_{1}}{d y}=\xi_{2} \frac{d f_{2}}{d y}
$$

the TE and TM polarisation parameters $\xi_{j}$ are defined in (5). The general solution for the field distribution $f_{j}^{N}(y)$ in the $j$-th layer of the $N$-th period is a superposition of the two field expressions in (1), and can be written in the form: 

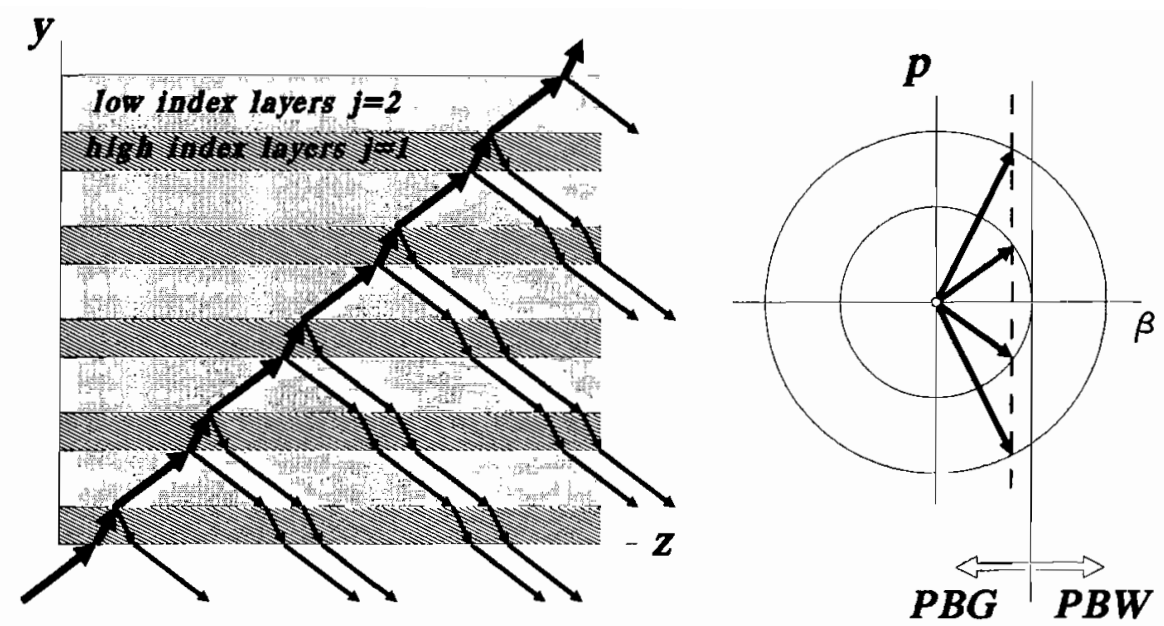

Figure 5. Geometry of multi-layer stack $\left(n_{1}>n_{2}\right)$, and the related wavevector diagram. The directions of the rays in each layer are easily predicted using the wavevector diagram at constant $\beta$. Under the first Born approximation, the reflections at each interface are very weak, the incident ray can be assumed undepleted, and single scattering dominates. The primary ray is illustrated with a somewhat thicker line.

$$
f_{j}^{N}(y)=a_{j}^{N} \cos \left[p_{j}\left(y-y_{j}^{N}\right)\right]+b_{j}^{N} \frac{\sin \left[p_{j}\left(y-y_{j}^{N}\right)\right]}{\xi_{j} p_{j} \Lambda},
$$

where $a_{j}^{N}$ and $b_{j}^{N}$ are arbitrary constants, $y_{j}^{N}$ is the value of $y$ at the centre of the $j$-th layer of the $N$-th period, and $p_{j}$ was defined in (2). In departure from previous treatments (e.g. reference 29), the functions in (20) have been carefully chosen for algebraic convenience. They are entirely real for all real values of $\beta^{2}$ even if $p_{j}$ is imaginary, are well-behaved as $p_{j}^{2}$ changes sign, and retain two degrees of freedom in the special case $p_{j}=0$. Also, waves within a stop-band are specified by entirely real values of the two constants $a_{j}^{N}$ and $b_{j}^{N}$, which have the same units. A two-component state vector made up of these constants completely specifies the field in the stack. The state vector in one layer can be expressed in terms of the state vector in the corresponding layer in the previous period by operation with a $2 \times 2$ translation matrix:

$$
\left(\begin{array}{l}
a_{j}^{N+1} \\
b_{j}^{N+1}
\end{array}\right)=\mathbf{M}\left(\begin{array}{l}
a_{j}^{N} \\
b_{j}^{N}
\end{array}\right) .
$$

By expressing the fields in (20) with respect to a local origin in the centre of a layer (instead of, for example, at the edge), the symmetry of the structure is maximally exploited, leading to a matrix $\mathbf{M}$ with eigenvalues and eigenvectors in a particularly simple form:

$$
\mathbf{M}=\left(\begin{array}{ll}
A & B \\
C & A
\end{array}\right),
$$

where the elements are given in Appendix A1. The eigenvalues and eigenvectors are: 


$$
\begin{aligned}
& \lambda_{ \pm}=A \pm \sqrt{B C} ; \\
& \left(\mathbf{f}_{ \pm}\right)=\left(\begin{array}{c}
\sqrt{B} \\
\pm \sqrt{C}
\end{array}\right),
\end{aligned}
$$

where $B C=A^{2}-1$ and $\operatorname{det}[\mathbf{M}]=1$, i.e., $\mathbf{M}$ is unimodular. This implies that the product of the eigenvalues $\lambda_{+}$and $\lambda_{-}$is unity, so that they can without loss of generality be written as

$$
\lambda_{ \pm}=\exp \left( \pm j k_{y} \Lambda\right)
$$

where $k_{y}$ is to be determined. Thus the state vector of each component field satisfies

$$
\left(\begin{array}{l}
a_{j}^{N+1} \\
b_{j}^{N+1}
\end{array}\right)=\left(\begin{array}{l}
a_{j}^{N} \\
b_{j}^{N}
\end{array}\right) \exp \left( \pm j k_{y} \Lambda\right) .
$$

The general field in the structure (for given $\omega, \beta$ and polarization) is expressible as a superposition of two Bloch waves with field distributions:

$$
f_{ \pm}(y) \mathrm{e}^{-j \beta z}=B_{ \pm}(y) \exp \left[-j\left(\beta z \pm k_{y} y\right)\right],
$$

where the function $B_{ \pm}(y)$ is periodic with period $\Lambda$ and the Bloch wavevector $k_{y}$ is given by:

$$
k_{y}=\frac{\cos ^{-1} A}{\Lambda} .
$$

If $k_{y}$ is real, the Bloch waves in (27) are progressive and may transport energy normal to the layers as well as along them. If, however, values of $\omega$ and $\beta$ exist for which the magnitude of $A$ exceeds 1 (or $B C<0$ ), $k_{y}$ has an imaginary part. The Bloch waves are then evanescent, growing or decaying exponentially from period to period normal to the layers, while progressing along them. If the structure is infinite (in the $y$-direction), these waves cannot be supported and no real states exist; the ranges of $\omega$ and $\beta$ where this occurs are the photonic band gaps. In a truncated structure, however, they play the role of tunnelling fields; for example, for incidence of a travelling plane wave, the stack behaves as a familiar multi-layer reflector. If the external field is itself evanescent, and matches to a Bloch wave decaying into the stack, a photonic surface wave can form (see section 9.1). The band edges between real and virtual states (i.e., travelling and evanescent Bloch waves) occur when $A= \pm 1$; an equivalent condition is $B C=0$.

Each Bloch wave can alternatively be expanded in terms of an infinite number of plane waves whose wavevectors are related by Floquet's theorem:

$$
\mathbf{k}_{n}=\beta \hat{\mathbf{z}}+\left(k_{y}+n K\right) \hat{\mathbf{y}}
$$

where $K=2 \pi / \Lambda$ is the reciprocal lattice wavevector (or grating vector). This permits us to express $B_{ \pm}(y)$ in the general form:

$$
B_{ \pm}(y)=\sum_{n} S_{n}^{ \pm} \exp (-\mathrm{j} n K y)
$$


where the $S_{n}^{ \pm}$are the complex plane wave amplitudes. Equations (28) and (29) may be used directly to plot the Brillouin and wavevector diagrams for the structure, as is done in later sections.

\subsection{Normalised Parameters}

An appropriate set of normalised parameters for the multi-layer stack treated above includes just three normalised parameters: a normalised frequency $\nu$, an index ratio $n_{R}$ and an relative thickness $\tau$ :

$$
v=k n_{a v} \Lambda, \quad n_{R}=\left(n_{2} / n_{1}\right), \quad \tau=\left(h_{2} / \Lambda\right),
$$

where $n_{a v}$ is the weighted average index, defined by:

$$
n_{a v}=\left(n_{1} h_{1}+n_{2} h_{2}\right) / \Lambda
$$

and the indices of the layers are $n_{1}>n_{2}$. For convenience, a normalised version of $\beta$ will occasionally be used, in the form

$$
b=\beta \Lambda \text {. }
$$

As we shall sometimes discuss electrons in a stack of alternating high and low potentials $U_{2}$ and $U_{1}$, the following definition of average potential will be useful:

$$
U_{a v}=\left(U_{1} h_{1}+U_{2} h_{2}\right) / \Lambda \text {. }
$$

In the next three sections, we use the above analytical expressions for $k_{y}$ to explore the behaviour of Bloch waves in singly periodic structure as a function of frequency and $\beta$. In section 4 , Brillouin diagrams for electrons and photons at $\beta=0$ are discussed, together with the physical origins of the band gaps and some other issues. In section 5 , the wavevector diagram at constant $\omega$ and variable $\beta$ is introduced, and in section 6 the behaviour at oblique incidence treated.

\section{SINGLY PERIODIC STRUCTURES AT NORMAL INCIDENCE $(\beta=0)$}

\subsection{Brillouin Diagrams}

The Brillouin diagram ${ }^{23,24}$ for electrons is a plot of all the permitted real wavevectors as a function of electron energy. The photonic equivalent is a plot of wavevector $k_{y}$ as a function of optical frequency. These diagrams are plotted in Figure 6 for normal incidence in a particular case (oblique incidence is treated in section 6). For very weak modulation $\left(n_{1} \simeq n_{2} \simeq n_{a v}\right.$ and $U_{1} \simeq U_{2} \simeq U_{a v}$ ), the diagram reduces to a series of parabolae for electrons:

$$
\begin{aligned}
h_{e}=\frac{2 m_{e} \Lambda^{2}}{\hbar^{2}} H_{e} & =u_{a v}+\left(k_{y}+2 n \pi\right)^{2} \\
u_{a v} & =\frac{2 m_{e} \Lambda^{2}}{\hbar^{2}} U_{a v}
\end{aligned}
$$

where $h_{e}$ is the normalised total electron energy, and to a series of straight lines of slope \pm 1 for photons: 

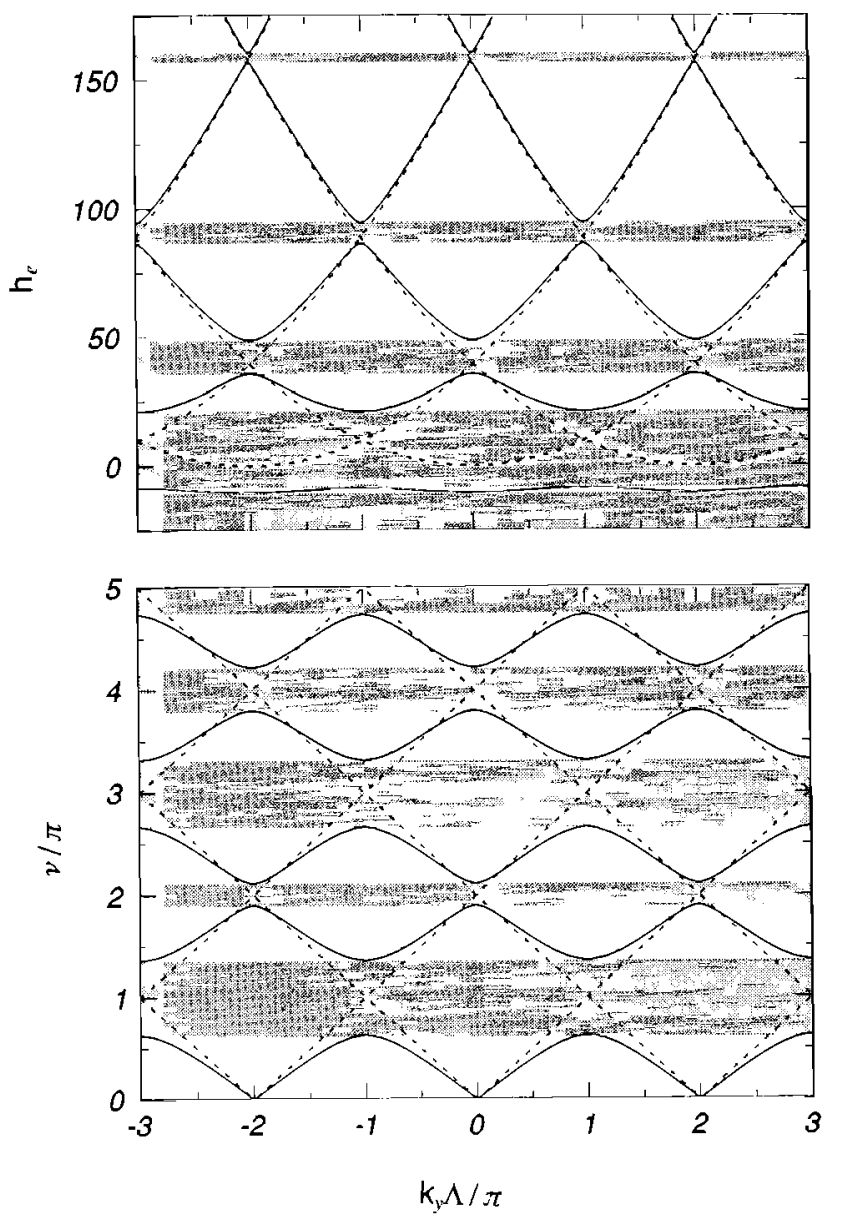

Figure 6. Brillouin zone diagrams for $\beta=0$ (normal incidence) on a multi-layer stack for electrons (upper) and photons (lower). The dotted lines are asymptotes, corresponding to a structure with the same average properties but a very weak modulation. In the electron case, $\tau=0.5, u_{2}=25$ and $u_{1}=-25$. In the photon case, $\tau=0.741$ and $n_{k}=0.286$ and the integers on the $v / \pi$ axis correspond to the Bragg conditions; the band gaps are all of comparable width (because "potential" and "total" energies both scale with frequency), and the asymptotes straight lines as expected. In the electron case, the band gaps narrow with increasing $h_{e}$, and the asymptotes are parabolic; note the appearance of a band window - a region where propagation is unexpectedly allowed - below the base of the parabolae. 


$$
v= \pm\left(k_{y} \Lambda+2 n \pi\right)
$$

The integer $n$ in each case refers to the $n$-th plane wave in the expansion in (30). When the single-pass optical path length across a unit cell, calculated using the components of wavevector $p_{1}$ and $p_{2}$ normal to each layer, is equal to an integral number $m$ times $\pi$, the $m$-th order Bragg condition is satisfied:

$$
p_{1}(1-\tau) \Lambda+p_{2} \tau \Lambda=m \pi \text {. }
$$

This condition originally arose in x-ray diffraction ${ }^{59-63}$, where the modulation depth of the periodic scattering potential is very weak, and in fact it assumes that the first Born approximation holds, i.e., that each interface contributes only a very weak reflection and that secondary and higher order reflections are negligible (see Figure 5 for an illustration of this). This is clearly not the case in a photonic band gap structure with very high modulation depths, when the Bragg condition becomes uncertain, spreading out over a range of frequencies (as discussed in section 4.4 below). Thus band gaps form at the intersections of the parabolae and straight lines, and a band window appears (for the parameters chosen) in the normally forbidden energy range $H_{e}<U_{a v}$ for electrons (Figure $6)$.

\subsection{Group Velocity and Density of States}

Just as for electrons in a finite crystal, the photonic states in a finite periodic structure are quantized (although they may be smeared out by strong coupling to the outside world). The density of states becomes large at the band edges since the wavevector $k_{y}$ there changes rapidly with frequency; states that would appear within the band gap if no periodicity were present are pushed to higher and lower frequencies, where they cluster in large numbers at the band edges. By reducing the density of states to zero within the band gap, the formation of photons is blocked, which can be used to suppress an unwanted radiative electronic transition ${ }^{7}$. The zero slope at the band edges implies that the group velocity vanishes, which makes sense since within the band gap photons cannot travel. There is of course a reciprocal relationship between density of states and group velocity ${ }^{23}$; however, unlike in the electron case when two electrons are permitted per state, each state can accommodate as many photons as desired (before the material becomes nonlinear or breaks down).

\subsection{Expectation Values of Potential: Electrons and Photons}

It will be useful, as preparation for the discussion of the origin of band gaps in section 4.4, to have supplemented the Brillouin diagram with plots of the expectation value of potential for both electrons and photons in a simple stack of alternating layers of high and low potential; this quantity varies according to how strongly the photons/electrons are redistributed within the unit cell. It also provides graphic illustration of some of the essential similarities and differences between electrons and photons in a periodic potential. For simplicity, normal incidence $(\beta=0)$ is assumed in each case, rendering the photonic solutions for TE and TM polarization identical. To facilitate the comparison, the wave equation is recast in a one-dimensional form common to electrons and photons:

$$
\left[-\Lambda^{2} \frac{d^{2}}{d y^{2}}+u(y)\right] \psi=e \psi
$$


where the normalised potential energy $u(y)$ is given by:

$$
u(y)=\frac{2 m \Lambda^{2}}{\hbar^{2}} U(y) \text { or }-v^{2}\left\{\epsilon_{r}(y)-1\right\} / n_{a v}^{2}
$$

and the normalised energy eigenvalue $e$ by:

$$
e=h_{e} \text { or } v^{2} / n_{a v}^{2}
$$

for electrons and photons respectively. The expectation value of $u(y)$ in each case is:

$$
\langle u\rangle=\frac{\langle\psi|u| \psi\rangle}{\langle\psi \mid \psi\rangle}
$$

where the averaging is carried out over a unit cell. Inside the stop-bands, where the fields
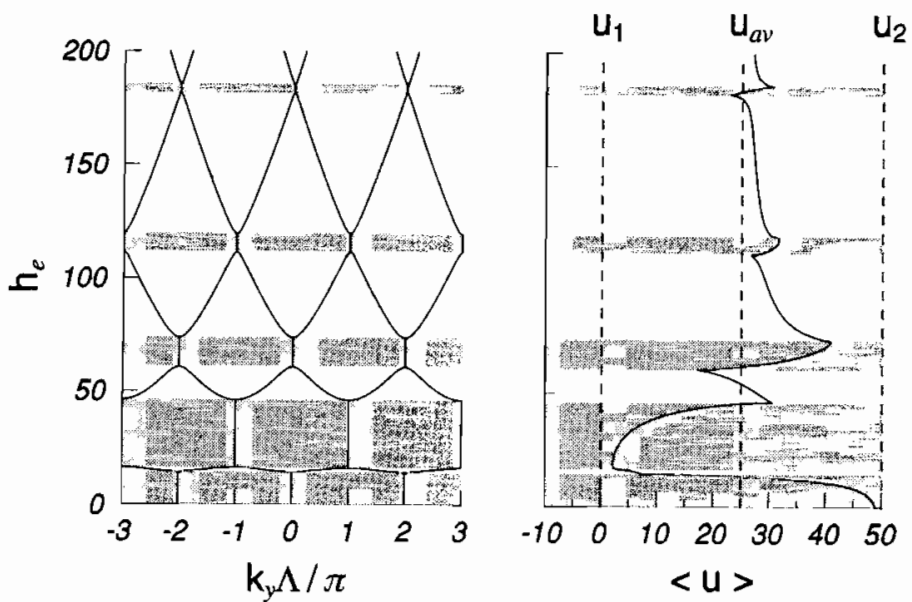

Figure 7. Wavevectors $k_{y} \Lambda / \pi$ and expectation values of potential energy $\langle u\rangle$ for electrons, plotted against energy eigenvalue $h_{e}\left(\tau=0.5, u_{1}=0\right.$ and $\left.u_{2}=50\right)$. The upper and lower bounds of $\langle u\rangle$ are constant ( $\propto$ lattice potentials). Note that $\langle u\rangle$ is consistently low on the low $e$ side of the band gaps, that the effect of the periodic potential diminishes with increasing total energy, leading to $\langle u\rangle \rightarrow u_{a v}$ at high energies.

are evanescent, expectation values cannot be calculated (the field grows/decays from cell to cell) because there are no real states; however, in the interests of following the redistribution of photons, the fields $\psi$ in (41) are multiplied by $\exp ( \pm \alpha y)$, where $\alpha=$ $\operatorname{Im}\left(k_{y}\right)$ is the evanescent decay rate of the Bloch wave. This compensates for the exponential growth/decay of the field from cell to cell.

First, for electrons, the Brillouin diagram is plotted in the form $h_{e}$ versus $k_{y} \Lambda / \pi$ (Figure 7). When the total energy $h_{e}$ is less than $\langle u\rangle$, the waves are evanescent. The 
lower and upper bounds on $\langle u\rangle$ are given by the minimum and maximum potential energies present in the lattice: $u_{1}$ and $u_{2}$ respectively. For total energies $h_{e}<u_{2}$, the tight binding approximation holds, and substantial variations in $\langle u\rangle$ occur as the electrons shift between regions of high and low potential energies. As $h_{e}$ increases, the number of cycles of field within the layers increases, and $\langle u\rangle$ tends towards the mean value $u_{a v}$. The expectation potential is consistently lower on the low energy band gap edges, and higher on the high energy band gap edges. At higher electron energies, the presence of the periodic potential becomes less and less important, and the fluctuations in $\langle u\rangle$ smaller and smaller; this reflects the fact that $u(y)$ is independent of $e$ for electrons.

Second, for photons, the upper and lower bounds of the potential scale with the
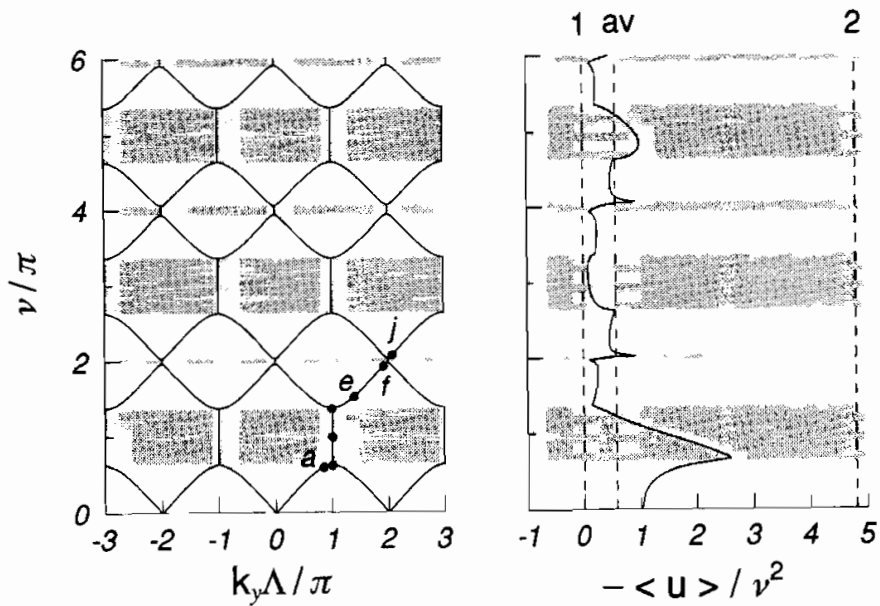

Figure 8. Wavevectors $k_{y} \Lambda / \pi$ and expectation values of the dielectric susceptibility $\left\langle\epsilon_{r}-1\right\rangle / n_{a v}{ }^{2}$, plotted against $\nu / \pi\left(\tau=0.7878, n_{1}=3.5\right.$ and $\left.n_{2}=1\right)$. The upper and lower bounds of $\left\langle\epsilon_{r}-1\right\rangle$ are $\left(n_{1}{ }^{2}-1\right)$ and $\left(n_{2}{ }^{2}-1\right)$ respectively. Note that, unlike in the electron case, $\left\langle\epsilon_{r}-1\right\rangle$ is not consistently low on the low $e$ side of the band gaps. The microscopic field intensity profiles for the five marked points ( $a$ to $e$ from low to high frequencies) are available in Figure 9 , together with the profiles for five similar points ( $f$ to $j$ - not marked in owing to lack of space) around the secondorder band gap at $\nu / \pi=2$.

optical frequency $(\propto k)$, so it is more convenient to plot $\left\langle\epsilon_{r}-1\right\rangle$, which we normalise to $n_{a v}{ }^{2}$; for consistency with Figure 6 the quantity $e$ is replaced with $\nu / \pi$. The diagram (Figure 8 ) is very different; unlike in the electronic case, tight binding and nearly free energy regions cannot co-exist on the same diagram, since the ratio between potential and total energy does not alter with increasing $\nu$. Note that for photons the expectation value of dielectric constant does not change consistently from high to low across the band gaps. Illustrative field intensity profiles across the unit cells for different points on the diagram are given in Figure 9.

We note in conclusion that a completely different comparison may be made at constant optical frequency (i.e., $k=$ constant). Under these circumstances, a diagram formally identical with the electron case in Figure 7 may be obtained for photons when 
$\beta>0$, in which case for TE polarization (38) can be rearranged as:

$$
\left[-\Lambda^{2} \frac{d^{2}}{d y^{2}}+u^{\prime}(y)\right] \psi=e^{\prime} \psi
$$

where $u^{\prime}=-k^{2} \epsilon_{r}$ and $e^{\prime}=-\beta^{2}$. Under these circumstances the "potential energy" is independent of the "total energy". We emphasise that this makes use of a completely different analogy to that in section 2 and the rest of this section.
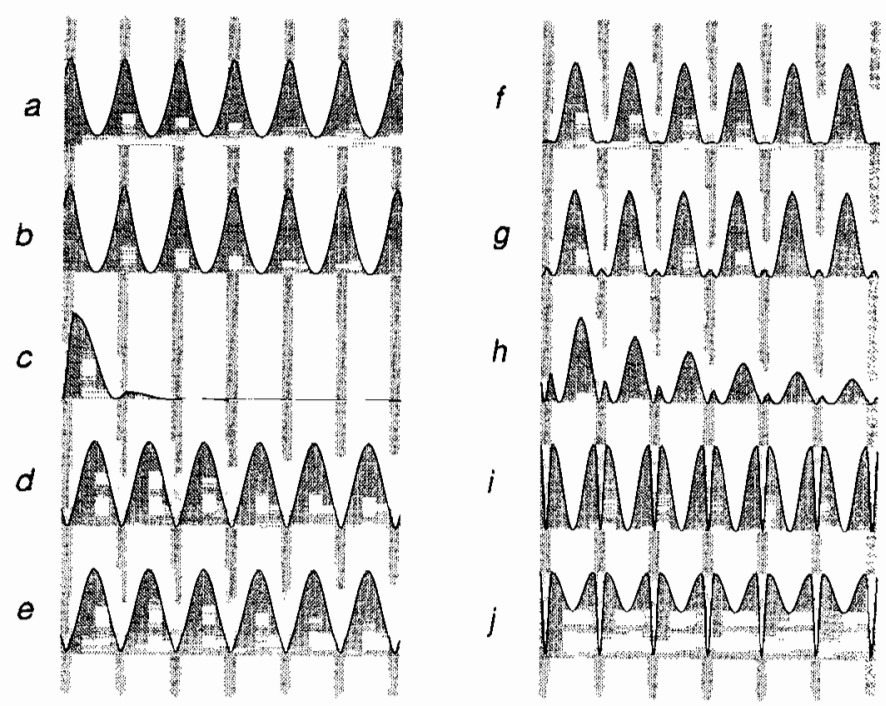

Figure 9. Field intensity distributions at points in Figure 8, as the first and second order Bragg conditions are traversed $(a$ to $j$ ). The redistribution of photons into high and low index regions gives rise to frequency-dependent expectation values of dielectric constant (also Figure 8), and can be used to explain to appearance of a photonic band gap.

\subsection{Origins of Band Gaps}

The accepted characteristic of a band gap is that it appears in regions where propagation is normally allowed (although see section 6.2 ). This statement is equally true of electrons in a semiconductor and photons in a PBG material. It is interesting at this point to ask why electrons in the valence band have restricted mobility (proportional to the reciprocal of the effective mass), while photons in a photonic "valence" band are free to move. The essential reason is that for photons the potential and total energy both scale with frequency; thus for $\beta=0$ it is not possible to move from nearly free (conduction) to tightly bound (valence) photons by changing the total energy. If, however, the optical frequency is fixed and $\beta$ is varied instead, a perfect analogy with electronic band theory is obtained as pointed out above (in equation (42)).

Perhaps the simplest qualitative explanation of band gap formation starts with field microstructure. As the Bragg condition is approached, the periodic structure becomes resonantly imaged by the light ${ }^{5}$. This occurs as the wavelets reflected at successive grating 
planes become more and more in phase, allowing them to build up into a strong reflected wave which interferes with the incident wave to produce a periodic image. If this image is invariant as the light progresses through the grating, it is a picture of a Bloch wave. The band gap then arises through the interplay of two sometimes conflicting requirements: 1) the light must produce an image with the same period as the structure; and 2) it must achieve this despite being redistributed by interference into regions of high or low dielectric constant.

As the band edge is approached for normal incidence $(\beta=0)$ and the image becomes resonant with the periodic structure, the expectation value of dielectric constant $\left\langle\epsilon_{r}\right\rangle$ increases or reduces according to the position of the fringes relative to the grating planes (section 4.3; Figure 8). At exact Bragg incidence, $\lambda / 2 n_{a y}=\Lambda$, suggesting that a perfect image can form; however, if this were so, interference would push $\left\langle\epsilon_{r}\right\rangle$ away

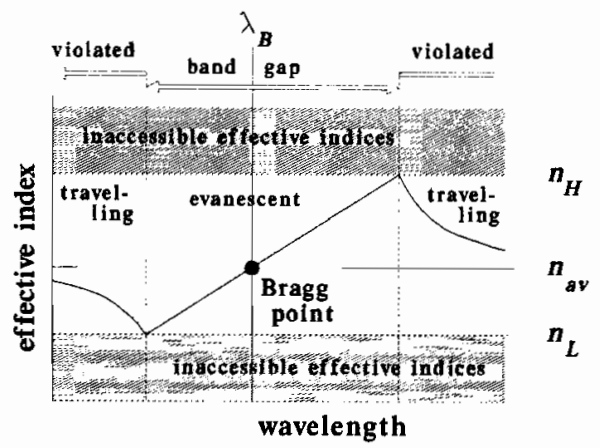

Figure 10. Diagram illustrating how wavelength-dependent changes in effective refractive index permit the Bragg condition to smear out over a band gap - a range of wavelengths limited by the degree to which photons are redistributed into high and low index regions.

to higher or lower values, thus altering the fringe period and ruining the image. What happens in practice is that the fringe pattern forms neither in nor out-of-phase with the grating, allowing $\left\langle\epsilon_{r}\right\rangle$ to reduce or increase until the correct period is found, i.e., until the real part of $k \sqrt{ }\left\langle\epsilon_{r}\right\rangle$ exactly equals $K / 2$. The penalty for this trickery is that the associated Bloch wave becomes evanescent - a consequence of the fact that a true image cannot form at an arbitrary phase to an object. Exactly on the band edges, it may be shown that $k \sqrt{ }\left\langle\epsilon_{r}\right\rangle=m K / 2$ where $m$ is the order of the Bragg condition; outside the band gap, moving away from the Bragg condition, fringes of the correct period are produced, but the image becomes increasingly less visible (this trend is apparent in Figure 9). Note that within the band gap, because the image is highly visible, the fields are zero at one or more points within each unit cell; this forbids energy flow across the layers, which is another way of stating that the group velocity normal to the planes goes to zero ${ }^{56,57}$. The position of the band edges can be crudely explained by generalising the first order Bragg condition 
to allow for wavelength-dependent changes in effective index $n_{\text {eff }}$ :

$$
\lambda=2 n_{\mathrm{eff}}(\lambda) \Lambda
$$

which is based on the notion that $\sqrt{ }\left\langle\epsilon_{r}\right\rangle$ is related in some monotonic (but not straightforward) manner to $n_{\text {eff }}$. This generalised Bragg condition is satisfied over a range of wavelengths given by the maximum and minimum values of $2 \Lambda \sqrt{ }\left\langle\epsilon_{r}\right\rangle$, yielding the positions of the band edges; the concept is illustrated in Figure 10. The fringes are out-ofphase with the grating at the high frequency band edge (photons concentrated in low index regions), and in-phase at the low frequency band edge (concentrated in high index regions). Note that these simple arguments are less useful at higher order Bragg conditions, when the field microstructure becomes much more complicated; indeed. the band gap can shrink to zero (see section 6.3) for a number of reasons.

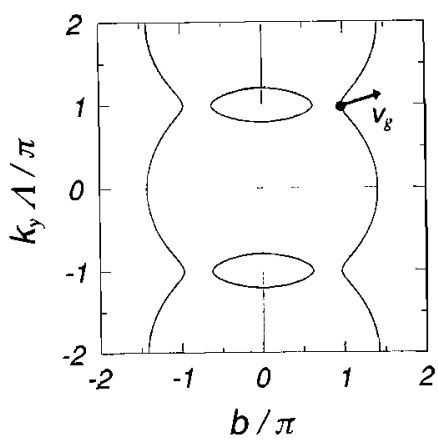

Figure 11. Example of wavevector diagram. The upper and lower shaded regions are higher order Brillouin zones, which are exact replicas of the first zone. A single point on a curve within the first zone brings with it all corresponding points within all the other zones; the wavevectors associated with these points are those of the complete set of plane waves needed to form a Bloch wave - see (30). The group velocity of the Bloch wave points in the direction of the normal (equation (44)).

\section{THE WAVEVECTOR DIAGRAM}

Most of the important features of two-dimensional propagation of photonic Bloch waves in periodic structures are usefully summarised on a wavevector diagram ${ }^{56}$, which is a plot of the locii of allowed real values of $k_{y}$ against $\beta$ for a given $\omega$ and polarization; it may be calculated using (28) and (29). A simple example is given in Figure 11. The resulting curves are known in $\mathrm{X}$-ray diffraction as dispersion surfaces ${ }^{61}$, and are related to constant energy surfaces in electronic band theory ${ }^{23}$. The diagrams are symmetric in $\beta$, periodic in $k_{y}$ because of the Floquet/Bloch theorem, and symmetric in $k_{y}$, since for every value of $k_{y}$ there is a value $-k_{y}$ corresponding to a Bloch wave progressing in the opposite 
direction. The diagram reveals the location of the stop-bands for a particular stack. A particularly useful feature of the diagram is its ability to predict the direction of the group velocity, via the relationship:

$$
\mathbf{v}_{g}=\nabla_{k} \omega(\mathbf{k})
$$

which shows that $\mathbf{v}_{\mathrm{g}}$ is oriented normal to the curves in wavevector space, pointing in the direction of increasing frequency (note that it ceases to have an obvious meaning for evanescent waves, when the wavevector is complex).

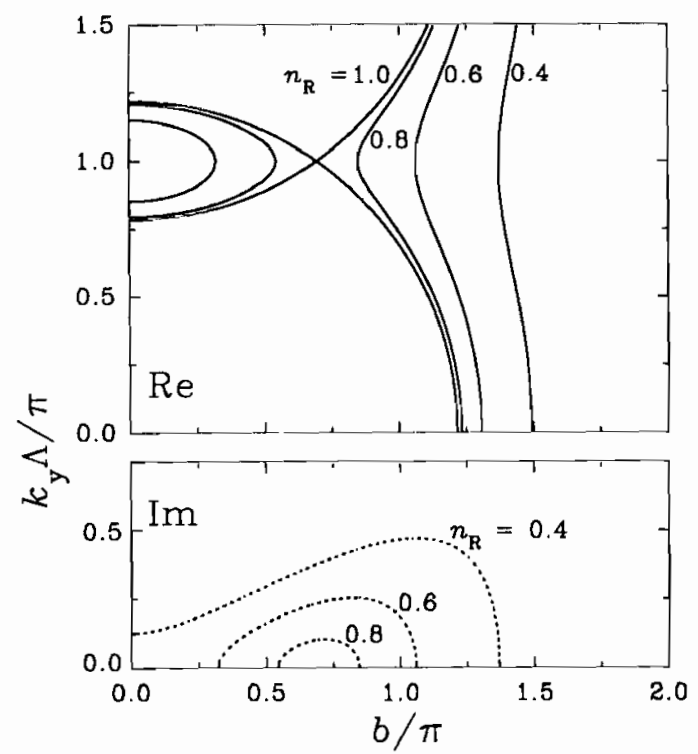

Figure 12. Portion of the wavevector diagram at $\nu=1.21 \pi$ for $n_{R}=1,0.8,0.6$ and 0.4 , keeping $n_{a v}$ constant by adjusting $\tau$ appropriately. At $n_{R}=0.4$ the left hand branch of the stop band has vanished and the right hand pass band has considerably narrowed. The imaginary parts of $k_{y} \Lambda / \pi$ for pure real $\beta$ are also plotted.

In Figure 12 the diagram is plotted for a variety of values of $n_{R}$. Notice how it is possible to suppress the inner (left hand) stop-band branch at high modulation depths (e.g., $n_{R}=0.4$ ), when the right hand stop-band branches become squashed-up and steep, lying well outside the circle corresponding to the average index in the stack (this is linked to the appearance of photonic band windows, and is discussed in more detail in section 6.2). For $\beta$ values within a stop-band, it is also instructive to plot the imaginary part of $k_{y}$, which gives the decay rate of the Bloch mode field in the $y$ direction.

It is of critical importance in optics to be able to predict what waves will be excited inside a periodic structure for an arbitrary incident wave. The wavevector diagram is ideal for this purpose; by superimposing the diagrams for each medium, and requiring the wavevector components tangential to the local boundary to be conserved, the complete set of travelling waves on each side of the boundary can be found ${ }^{57}$. The direction in which 
the light proceeds within the grating is then given by the normal to the dispersion surfaces. The method is described in more detail in section 7.2, where it is used extensively in the discussion of devices and two-dimensional propagation.

\section{SINGLY PERIODIC STRUCTURES AT OBLIQUE INCIDENCE $(\beta>0)$}

In this section, the effect of oblique incidence $(\beta>0)$ on the band gap widths and positions is explored. This is an important issue if singly periodic stacks are to be used in the control of spontaneous emission in lasers ${ }^{37-40}$ (see also other chapters in this volume). In the ensuing sub-sections, band-edge diagrams are discussed, the idea of a photonic band window introduced, the conditions causing the PBG to shrink to zero identified, and finally the Brillouin diagram at $\beta=$ constant is used to illustrate how under these conditions photons can resemble electrons.

\section{- 6.1 Band-Edge Diagrams}

It is useful to know in detail how light will behave in frequency regions outside the main photonic band gap. For example, although the creation of a photonic band gap may successfully suppress an intermediate radiative transition in an up-conversion laser, higher order band gaps could, if not properly understood and controlled, interfere with the laser's performance. For this and many other reasons, it is useful to plot the band edge positions on a diagram of normalised frequency versus $\beta$. The band edges are located at points where the product of off-diagonal matrix elements is zero, i.e., $B C=0$ in (23). The resulting diagram (Figure 13) divides up into three main regions: a photonic band gap region where free propagation is normally expected but gaps appear; a photonic band window region where the norm is evanescence but windows appear (the light being tightly bound inside each high index layer); and a cut-off region where propagation never occurs. The first two of these regions have analogies in the nearly free electron and tight binding models of electronic band theory ${ }^{23,24}$.

\subsection{Photonic Band Gaps and Windows}

Two complementary views of photonic band structure are possible: It is either a sequence of band gaps in a frequency range where light is otherwise free to propagate; or a sequence of band windows in a frequency range where light is otherwise localised, i.e., evanescent. Which of these views is most appropriate depends on our understanding of the words "otherwise free to propagate" and "otherwise localised." One interpretation makes use of the average index; however, as already mentioned, it is difficult to assign this a useful value owing to redistribution of photons into regions of high and low dielectric constant (for example, the multi-layer stack whose one-branch wavevector diagram is given in Figure 12 with $n_{R}=0.4$ can be viewed as a material with a refractive index that depends strongly on angle). Even so, some qualitative observations based on refractive index may be made. It is clear that tight binding cannot exist in the range $0<\beta<k n_{2}$, where the light is free to propagate in both layers; this is definitely a photonic band gap region. If, however, $\beta$ exceeds $k n_{2}$, the light in the low index layers is evanescent, and each high index layer will support a micro-resonance (a waveguide mode). The light is then able to progress across the layers by a process of resonant tunnelling ${ }^{52}$. As the value of $\beta$ increases, the light is more and more tightly bound inside each high index layer, and this tunnelling process becomes more difficult and slower; its efficacy depends on the width of the low index layers, i.e., the geometry of the stack. At infinite interlayer (atomic) spacing, the bound states correspond exactly to guided modes, with infinitely 

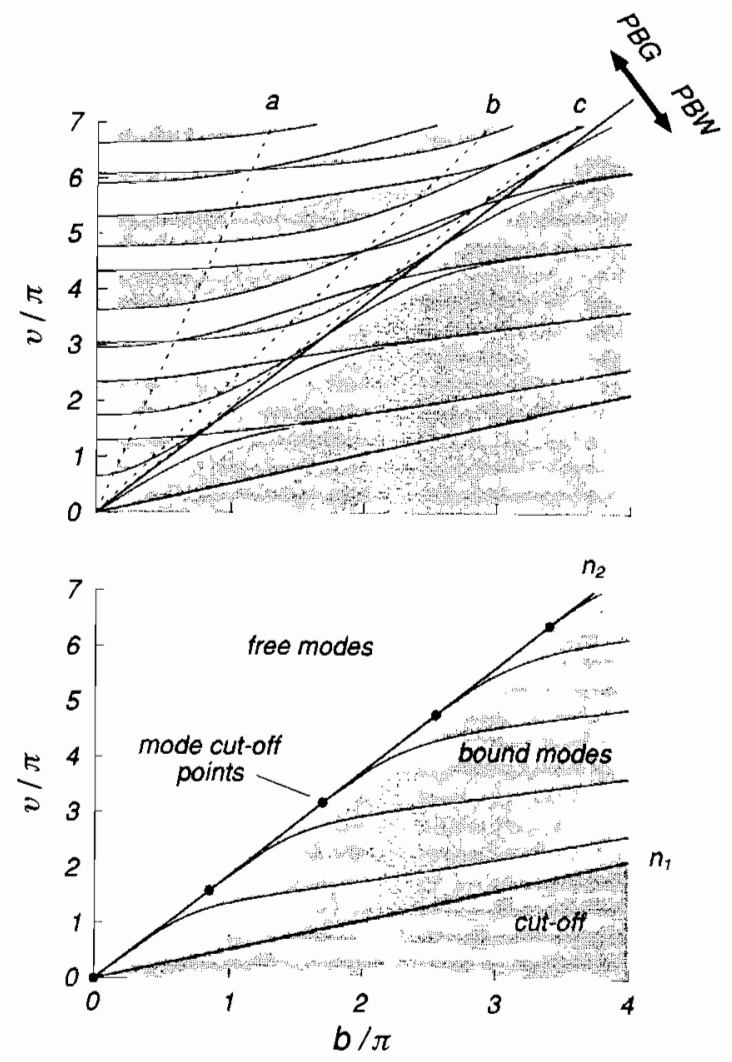

Figure 13. Upper: Band-edge diagram (TM polarization) in a multi-layer stack with $\tau=0.65$ and $n_{R}=0.286$; Lower: guided modes of a single high index layer; note free, bound (only points lying on the curves are permitted) and cut-off regions. Regions where propagation is forbidden are shaded. The higher order bound modes turn on at different values of $\nu / \pi$. In the stack, photonic band gaps (Bragg conditions) form in the free region and photonic band windows (resonant tunnelling between bound modes) form in the bound region. The transition occurs at $\beta=k n_{2}$. The band gaps shrink to zero at the Brewster condition (dotted line $c$ ), and at anti-resonances (two illustrative sequences indicated by the dotted lines $a$ and $b$ ); see section 6.3 for details.

sharp micro-resonant frequencies. As the interlayer spacing falls, the bound states begin to interact, their resonances smearing out over a range of frequencies to form a photonic band window. If the interlayer spacing is small so that considerable overlap exists between the photonic "orbitals," photons are relatively free to travel across the layers: the nearly- 

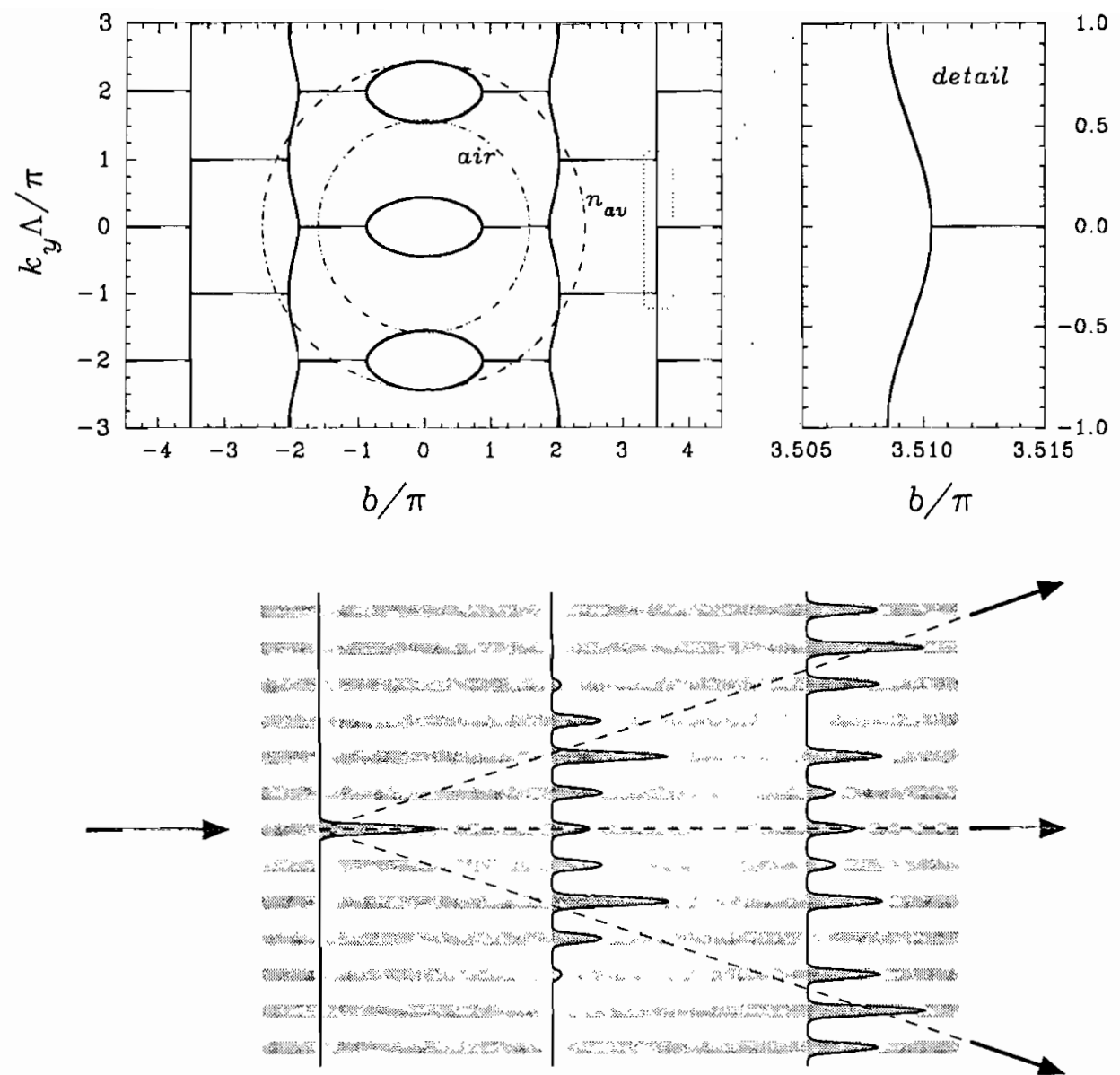

Figure 14. Upper (a): Wavevector diagram for $\tau=0.35, n_{R}=0.4, \nu=0.97 \pi$; resonant tunnelling between the high index layers is very slow in the tightly squashed-up pass band near $b / \pi=$ 3.5 , and less so in the band near $b / \pi=2$. Lower $(b)$ : A schematic diagram of the tunnelling process for excitation of a single "waveguide"; note that two pulses emerge above and below the initial guide, in the vicinity of which some light remains (group velocity is horizontal at $k_{y} \Lambda / \pi= \pm 1 \& 0$ ).

free photon approximation then becomes valid again.

In the tight-binding PBW regime, the group velocity direction (but not its magnitude) changes very little over each half of the Brillouin zone (Figure 14a). This creates, in optical terms, a highly anomalous situation where the phase velocity changes rapidly (thereby permitting good spatial resolution of small objects) while the group velocity is constant in direction (thereby avoiding Fresnel diffraction). This provides a physical basis from which to interpret an experiment on an array of parallel channel waveguides reported by Garmire et $\mathrm{al}^{52}$. In that experiment, light was coupled into a single central waveguide, and tunnelled sideways into the neighbouring guides; the waveguide array is thus "imaged" by the light, while the group velocity slowly carries the power sideways across the waveguides via resonant tunnelling (Figure 14b). An alternative interpretation of this experiment in terms of spatial Wannier functions is briefly introduced in section 7.2 .

Finally, we should like to mention the possibility of creating a PBW in a metal 
containing a periodic array of dielectric micro-cavities ${ }^{5}$. If these micro-cavities can "talk to each other," light will be able to tunnel through the metal in narrow frequency ranges around the resonant frequencies of the cavities. Alternatively described, a PBW will open up in regions of the spectrum where certain photonic states, their periodic field intensities peaking in the interstices between the metal walls of the cavities, are able to sneak through the structure without attenuation. In a real metal at optical frequencies, dissipation is likely to be a problem, but the concept is nonetheless valid.

\subsection{Points of Zero Band Gap Width}

It is important to understand the physical circumstances under which an otherwise wide photonic band gap can close up. Two different things can cause this to happen. The first is co-incidence of a Bragg condition (37) and the Brewster condition (3), which occurs when the rays in each layer are incident on the interfaces at Brewster's angle, reducing the reflection at each interface to zero; this happens only when the light is $p$-polarized (TM case). The second effect is more subtle, but just as simple; and it is even more important, since it occurs for both TE and TM polarized light. It happens when the anti-reflection condition discussed in section 1.2.5 applies concurrently to both layers; this occurs when the optical path lengths across each layer are separately a multiple of $\pi\left(m_{1} \pi\right.$ and $m_{2} \pi$ in layers 1 and 2, $m_{1}$ and $m_{2}$ being integers). At the same time, as stated mathematically in (37), the $m$-th order Bragg condition occurs when the single-pass optical path length across a unit cell is equal to $m \pi$. The points of zero stop-band width (Figure 13) therefore occur when both these conditions are simultaneously satisfied, i.e., when:

$$
\frac{p_{1} h_{1}}{m_{1}}=\frac{p_{2} h_{2}}{m_{2}}=\pi, \quad m_{1}+m_{2}=m .
$$

Solving these equations yields:

$$
b=\frac{\pi m_{1}}{1-\tau} \sqrt{\frac{\alpha^{2}-n_{R}{ }^{2}}{n_{R}{ }^{2}-1}}=v \sqrt{\frac{\alpha^{2}-n_{R}{ }^{2}}{\alpha^{2}-1}}
$$

where

$$
\alpha=m_{2}(1-\tau) / m_{1} \tau
$$

is used for convenience of notation. This corresponds to a situation where the total reflection from each unit cell is zero, i.e., when an anti-reflection resonance appears simultaneously in each layer. These points of zero stop-band width are much more common at higher frequencies, which explains why complete photonic band gaps are much more difficult (if not impossible) to find in higher order energy bands. In both cases,

$$
\mathbf{M}=\mathbf{M}^{\prime}= \pm\left(\begin{array}{ll}
1 & 0 \\
0 & 1
\end{array}\right)
$$

and the multi-layer structure behaves as a uniform medium with an average index different from $n_{a v}$ but no Bragg reflection. It may be possible to defeat (or at least mitigate) this effect by constructing more complicated unit cells with more than two different materials, such that the ratios of optical thicknesses are never simultaneously rational numbers. 


\subsection{Brillouin Diagrams for finite $\beta$}

We now turn our attention to the Brillouin diagram for photons under the slightly artificial circumstance when the momentum $\beta$ along the layers is constant but not zero (Figure 15). This form of oblique incidence turns out to be useful for illustrating how photons can become increasingly free as their total energy increases; the photonic states track along a vertical line in the band edge diagram (Figure 13). As the energy $H_{o}$ increases, the real photonic states move from being first cut off, to being concentrated in narrow band windows within an otherwise cut off region, to being permitted everywhere except within the photonic band gaps; the photons become increasingly less tightly bound.

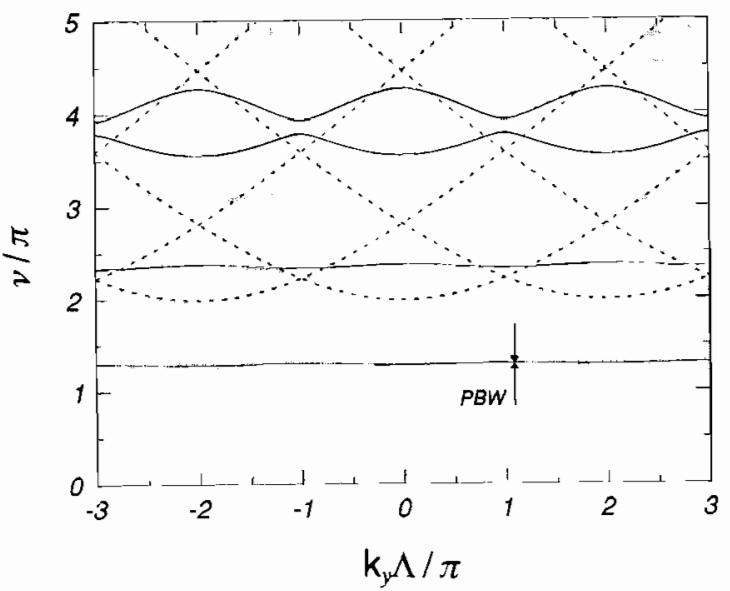

Figure 15. Brillouin diagram for photons at $b=\beta \Lambda=1.988 \pi$, with $\tau=0.741$ and $n_{R}=$ 0.286 (TE polarisation); the dotted lines are asymptotes, corresponding to a structure with the same average index but a very weak modulation $\left(n_{R} \rightarrow 1\right)$. The diagram is reminiscent of the electron case at normal incidence (Figure 6), although the asymptotes are this time hyperbolic with frequency; the pass bands widen with increasing frequency, and a band window opens up below the base of the hyperbolae (section 6.2).

As before, it is useful to plot the Brillouin diagram for the case when the scattering from each interface is vanishingly small; under these circumstances, the relationship between $\nu$, $\beta$ and $k_{y}$ for the $n$-th plane wave in the expansion (30) is given simply by:

$$
v=\sqrt{b^{2}}+\left(k_{y} \Lambda+2 n \pi\right)^{2} \text {. }
$$

Note that for $b^{2}>v^{2}$, the photons cannot normally (see section 6.2) propagate into the stack, although they will still progress along it with wavevector $\beta$. The appearance of real wavevectors within the cut-off region is intriguing, as it is reminiscent of the behaviour of electrons discussed in Figure 6. These wavevectors are caused, as discussed in section 6.2, by the excitation of sharp micro-resonances where the light is concentrated almost entirely 
within the high index layers. This permits a real wave to exist for combinations of energy and wavevector where, based on a mean index $n_{a v}$, evanescence would normally be expected.

\section{TWO-DIMENSIONAL PROPAGATION}

The aim in this section is to discuss the main features of two-dimensional propagation in singly periodic media, and to present some illustrative experimental results taken from work on periodic planar waveguides. First of all a Newtonian effective mass method is developed to handle the propagation of light in structures with a slowly varying average dielectric constant. Experimental results are then presented on this phenomenon, together with examples of refraction, diffraction and interference. Extensive use is made of the wavevector diagram introduced in section 5 . All the tools used can be extended without difficulty to multiply periodic structures whose wavevector diagrams are known.

\subsection{Effective Mass Method For Non-Uniform Periodic Structures}

Near a band edge, the effect of the lattice potential on the motion of an electron can be represented by replacing the electron rest mass with an effective mass $m_{e}{ }^{*}$. If the electron is subjected to an external force, its motion can then be modelled by Newton's laws for a particle with mass $m_{e}{ }^{*}$. This effective mass method is also known to be very useful in analysing the behaviour of electrons in non-uniform crystals containing slow variations in mean potential, such as can happen around dislocations, inclusions and other structural defects ${ }^{23,25}$. Under these circumstances, in the absence of an external electric field, the total electron energy is constant. We now develop an equivalent method for photonic Bloch waves in a periodic structure with a slowly varying average dielectric constant (the photonic equivalent of potential - see (12)), and illustrate it in the next section with experimental results on periodic planar waveguides. In both cases it is the curvatures of the $H-\mathbf{k}$ Brillouin diagram that determine the elements of the reciprocal effective mass tensor, via the formula stated in (13).

We have already seen in section 2.2 that there is a profound difference between the effective masses of electrons and photons in uniform isotropic media; for photons, a straightforward adaptation of (13) to the one-dimensional isotropic case leads to an effective mass of infinity, since the curvature of the $\omega-k$ diagram for free photons is then zero. Although this reflects the fact that photons cannot be accelerated or decelerated in a one-dimensional isotropic system, it is of little practical significance since to test it would require the creation of a force field for photons - something which is trivial for electrons (simply apply a voltage) but which is unknown for photons. As already pointed out (section 2.2), however, photons may be deflected, when they exhibit a finite effective mass of $2 m_{o} n$.

In periodic media, however, it turns out that electrons can acquire some of the properties of photons (e.g., very large effective mass in the direction of motion), and viceversa (e.g., finite effective mass in the direction of motion). In a PBG dielectric a slowly varying average dielectric constant at fixed optical frequency plays the same role for photons as an electric field does for electrons, permitting an effective mass to be defined and a Newtonian model of their motion to be constructed. Note that other forms of nonuniformity (pitch, refractive index difference) result in a variable effective mass and cannot be treated using this approach. At constant optical frequency, the "mass" $m_{o}$ and the total energy $H_{o}$ in (11) are constant, while the mean value of the potential $U_{o}$ is allowed to vary slowly. For formal simplicity, we restrict the analysis to the TE case when the wave equation is scalar. The Hamiltonian may then be written: 


$$
H_{o}=\frac{1}{2}\{\mathbf{p}\}^{\mathrm{T}}\left[1 / \mathbf{m}_{\mathbf{o}}^{*}\right]\{\mathbf{p}\}+W_{o}(\mathbf{r})
$$

where $\{\mathbf{p}\}$ is the momentum and $W_{o}$ is the slowly varying potential left behind after taking account of the periodic potential $U_{o}$ by using $m_{o}{ }^{*}$ rather than $m_{o}$ (i.e., the total potential is $W_{o}+U_{o o}$, where $U_{o o}$ is the mean value of $U_{o}$ ). Deriving Hamilton's equations of motion is now straightforward:

$$
\begin{aligned}
& \{\dot{\mathbf{p}}\}=-\nabla H_{0}=-\nabla W_{o}, \\
& \{\dot{\boldsymbol{\rho}}\}=\nabla_{\mathrm{p}} H_{o}=\left[1 / \mathbf{m}_{\mathrm{o}}^{*}\right]\{\mathbf{p}\}
\end{aligned}
$$

where $\{\boldsymbol{\rho}\}$ is the position vector and $\nabla_{\mathrm{p}}$ represents the gradient in momentum space. After differentiating the second equation and rearranging we obtain Newton's equations of motion with a force $-\nabla_{\mathrm{p}} W_{o}$ :

$$
\{\ddot{\boldsymbol{\rho}}\}=-\left[1 / \mathbf{m}_{\mathbf{0}}^{*}\right] \nabla W_{o}
$$

Notice that, owing to the tensor nature of the reciprocal effective mass, the acceleration is not necessarily in the direction of the changing potential.

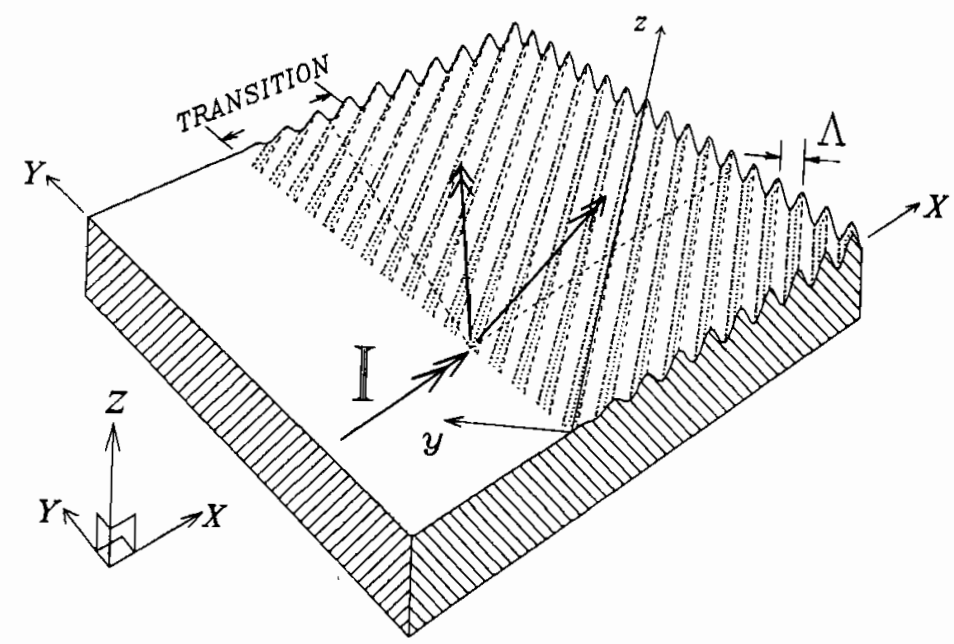

Figure 16. Sketch of the experimental set-up for exciting two-dimensional guided Bloch waves in a periodic planar waveguide ( $140 \mathrm{~nm}$ thick film of $\mathrm{Ta}_{2} \mathrm{O}_{5}$, on a glass substrate, with an etched corrugation of period $300 \mathrm{~nm}$ ). A guided mode is launched by prism coupling.

\subsection{Illustrations From Experiments On Planar Waveguides}

Periodic planar waveguides are ideal for studying Bloch wave propagation in two dimensions ${ }^{54-58}$. A sketch of the experimental set-up for excitation (by prism coupling) of a typical corrugated planar waveguide is given in Figure 16; a narrow beam is launched in the non-periodic guide region, travelling towards the periodic region, where it excites guided two-dimensional Bloch waves that can be observed (via the scattered light from the 

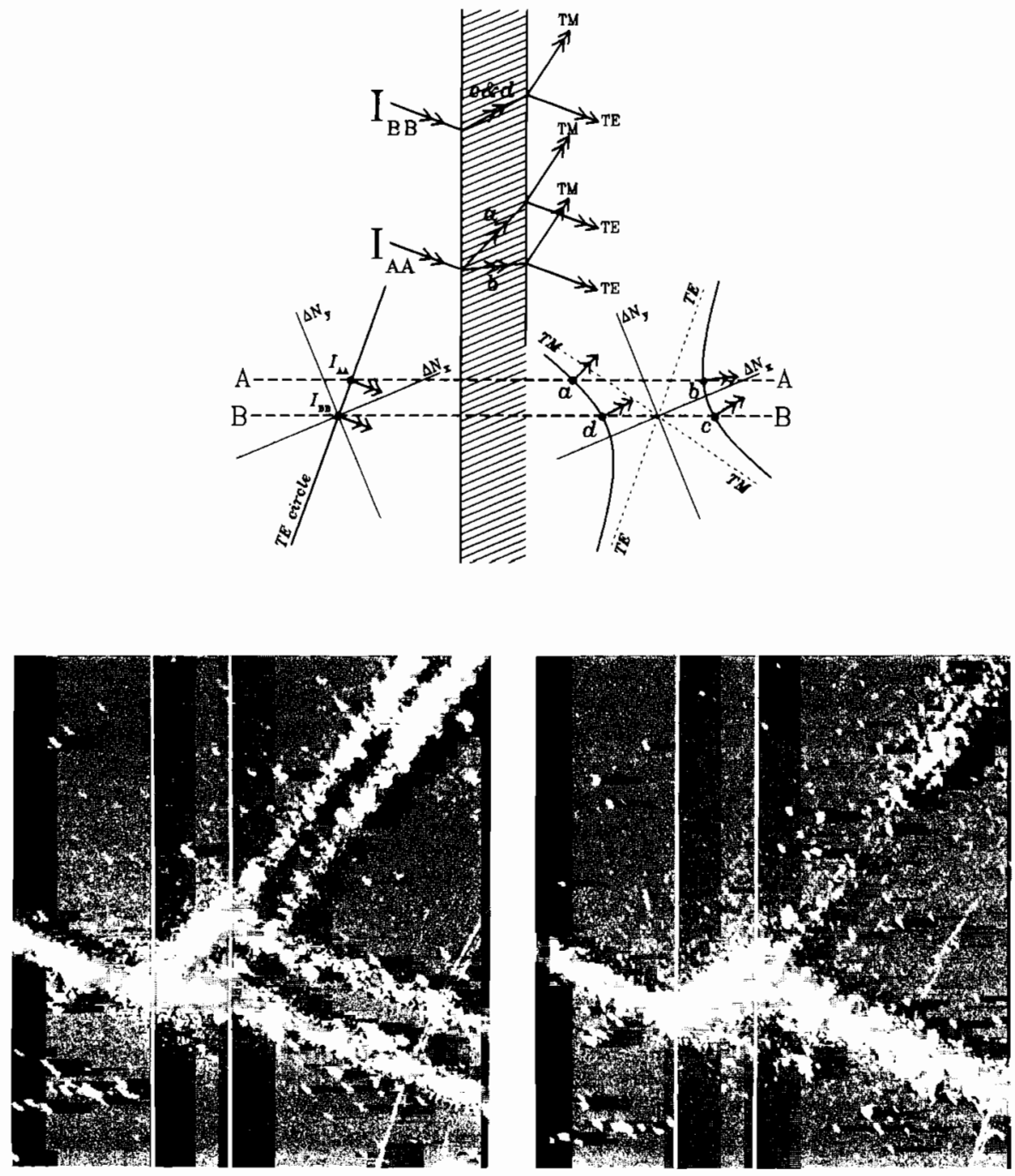

Figure 17. Upper: Double $\left(I_{\mathrm{AA}}\right)$ and single $\left(I_{\mathrm{BB}}\right)$ negative refraction at a parallel slab containing a singly periodic structure; note the use of the wavevector diagram to match the boundary conditions. Because the conversion is TE/TM, the asymptotic circles that cross at the stop-band centre have different radii; this causes an asymmetric stop-band. Lower: Photographs of the effect in a corrugated planar waveguide (slab width $1 \mathrm{~mm}$ ). The grating lines are slanted upwards to the right as shown schematically.

inevitably imperfect guide) by eye. In the experiments described in this section, the waveguide is formed from a layer $\sim 140 \mathrm{~nm}$ thick of $\mathrm{Ta}_{2} \mathrm{O}_{5}$ (index 2.12), deposited by if sputtering onto a glass substrate (index 1.472). For these parameters, solutions of (4) yield guided mode refractive indices of 1.775 (TE) and 1.569 (TM) at $632.8 \mathrm{~nm}$. The waveguides were etched with a periodic pattern to form a corrugated upper surface with 

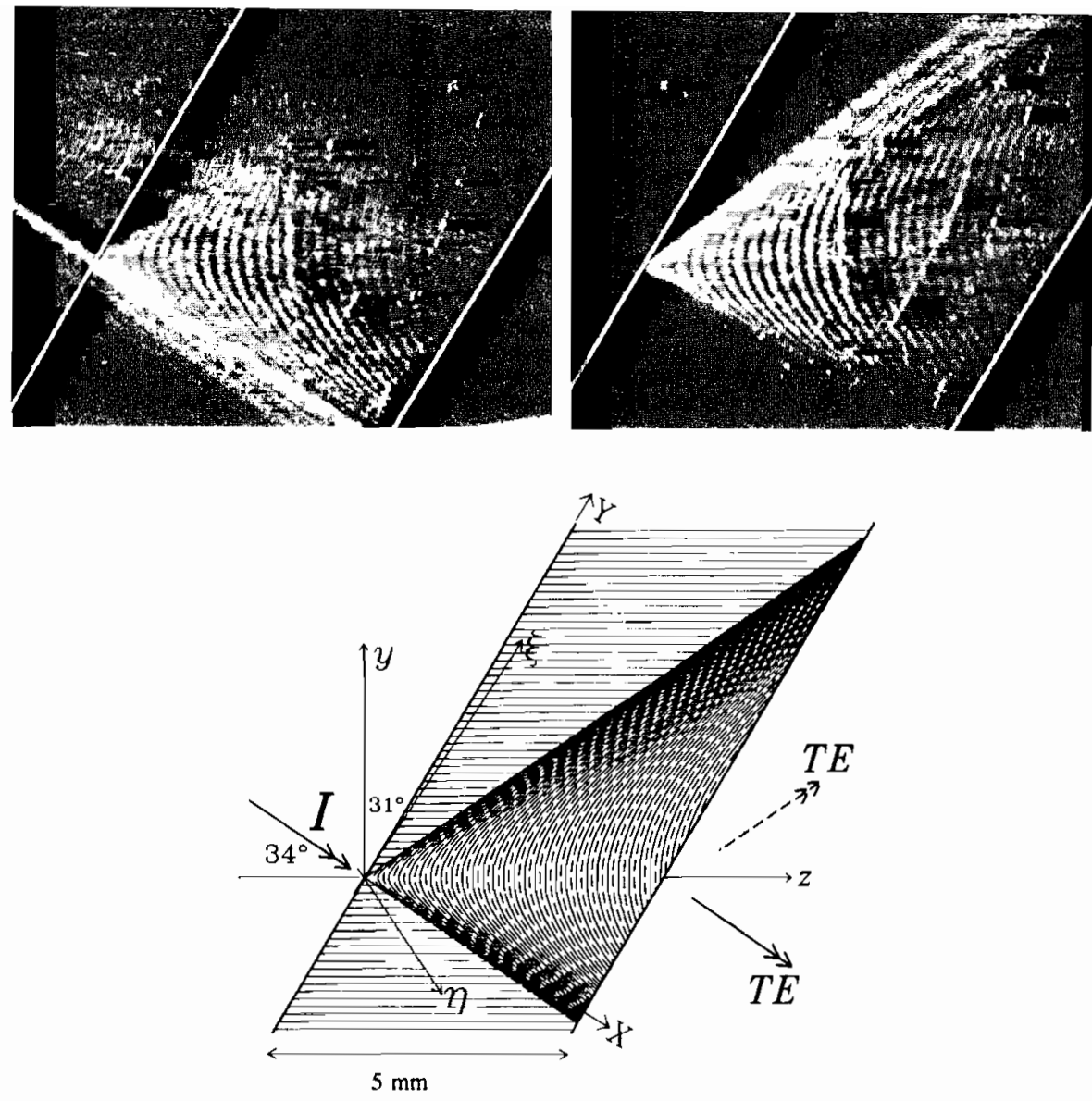

(a)

(b)

Figure 18. Point influence function for the fields in a singly-periodic medium (grating lines horizontal). Note how the power spreads out over a region bounded by the upper and lower Bragg angles (upper and lower edges of triangular region containing the fringes), and how interference occurs owing to spatial superposition of Bloch waves from opposite sides of the stop-band.

a pitch of $300 \mathrm{~nm}$.

Two things are needed to understand completely the propagation of a wave: its phase velocity (for boundary condition matching); and its group velocity (for predicting where it's going). Both are available simultaneously on the wavevector diagram for the periodic waveguide. As explained in section 5, the phase velocity is given by $\left(\omega /|\mathbf{k}|^{2}\right) \mathbf{k}$, where $\mathbf{k}$ is the vector between the origin of wavevector space and a point on the wavevector diagram; and the group velocity points normal to the curves, in the direction 
of increasing frequency (denoted by double-headed arrows in the figures in this section). Since the waveguide supports both TE and TM mndes, Bragg conditions exist for TE/TE, TE/TM, TM/TE and TM/TM modal coupling. In Figure 17 the collision of a narrow TEpolarized guided-beam with a parallel-sided periodic region is illustrated; the coupling is between TE and TM polarized guided modes. The beams incident from the uniform region have wavevectors that sit on the arc of the TE circle (points $I_{\mathrm{AA}}$ off-Bragg and $I_{\mathrm{BB}}$ onBragg in the figure). The Bloch waves excited within the grating are found by matching
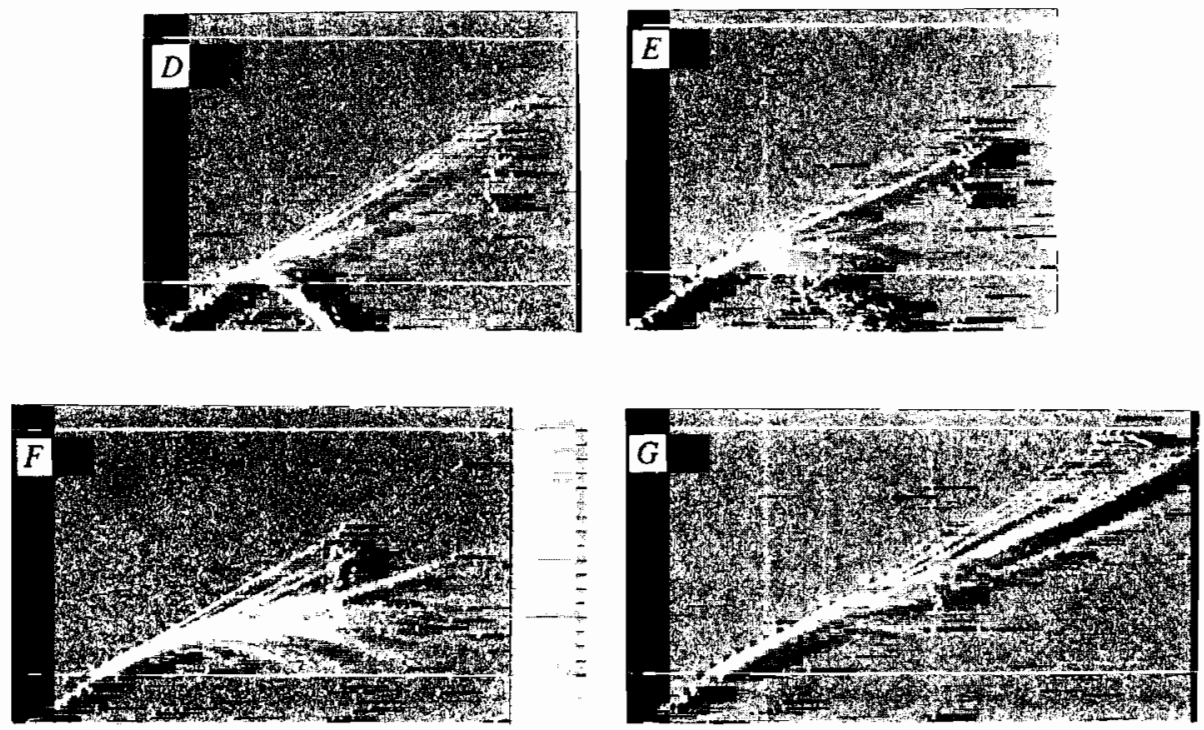

Figure 19a. Propagation of Bloch waves in grating with non-uniform average refractive index. In the experiment, $n_{a \nu}$ peaked in the centre of the parallel-sided periodic region $1.5 \mathrm{~mm}$ thick (see case $F$ for orientation of grating lines). The conversion is TE-TM. The cases $D-G$ are for different mean angles of incidence of a bundle of convergent rays. Notice the curved paths taken by the rays; the average index "hill" is viewed as a potential barrier by the light. See Figure $19 \mathrm{~b}$ for the results of an effective mass analysis.

wavevector components along the boundary. A simple graphical construction (similar to the one used in section 1.2) achieves this; construction lines AA and BB are drawn normal to the boundary, passing through points $I_{\mathrm{AA}}$ and $I_{\mathrm{BB}}$. These intersect with the stop-band branches in reciprocal space at the points associated with the Bloch waves excited inside the periodic structure. The normals to the stop-band branches at these points lie parallel to the group velocities of the Bloch waves, i.e., their rays. Notice that single negative refraction occurs on-Bragg (both Bloch waves sharing the same ray paths), and that double 

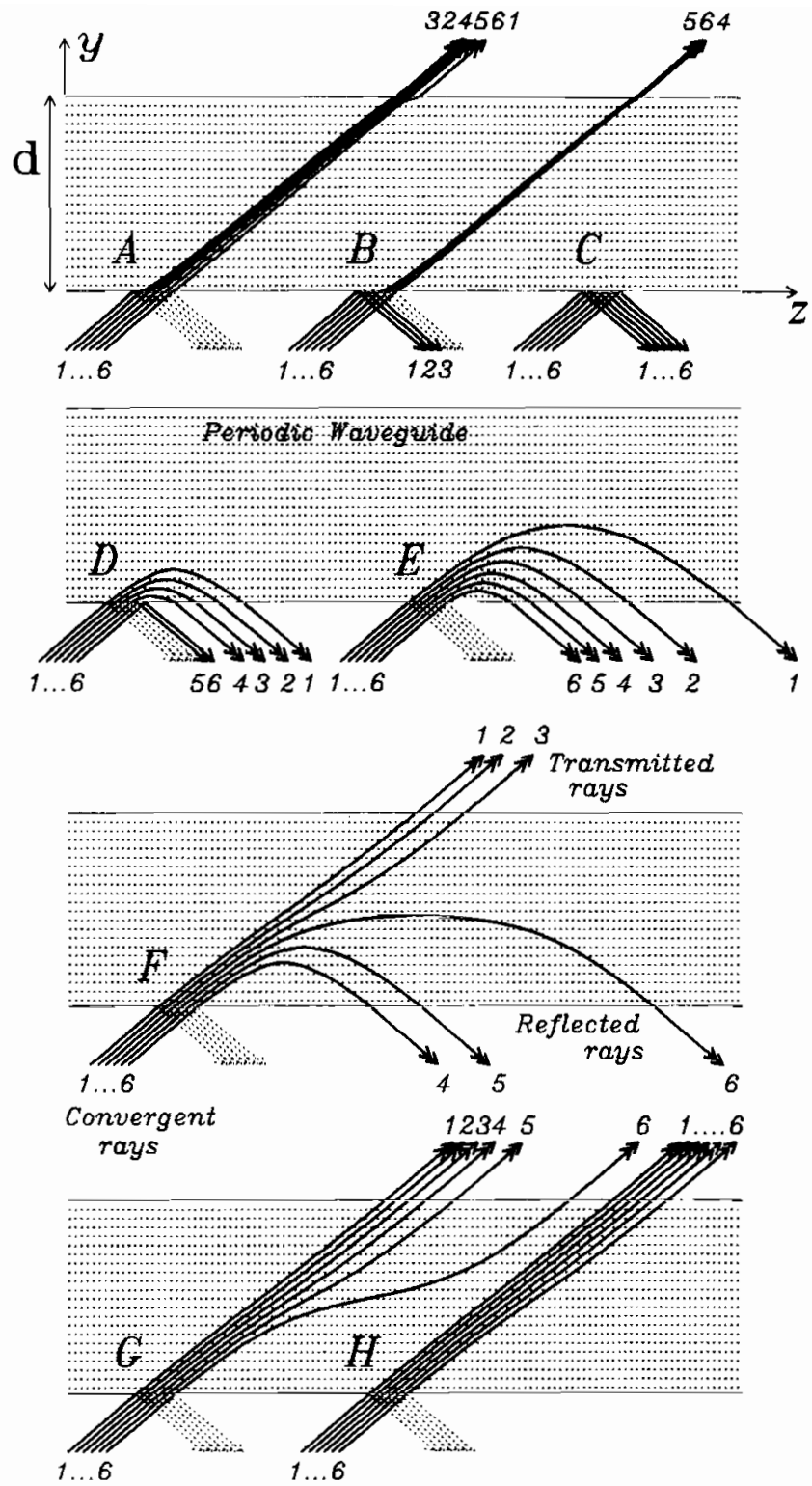

Figure 19b. The photographed behaviour in Figure 19a is qualitatively well predicted by a simple ray tracing procedure, based on the effective mass method described in the text. The sketches $A-H$ are for different mean angles of incidence of a bundle of convergent rays. Cases $D-G$ correspond most closely to the four experimental cases.

negative refraction occurs off-Bragg (each Bloch wave having a different ray path). Note that the behaviour is a sensitive function of the incident angle. At the output surface, the same boundary matching procedure yields the directions of the exit beams. 
When the incident beam is tightly focused, its angular plane wave spectrum can spread over the entire stop-band ${ }^{57,58}$. By analogy $w$ ith electronic band theory ${ }^{23,25}$, this type of excitation is approximately described by a kind of incomplete "spatial Wannier function", i.e., the integral of all the photonic Bloch states present in the stop-band portion of a Brillouin zone. In a weak singly periodic structure, these functions take the form of Bessel functions, and may be interpreted as point influence (Green's) functions (Appendix A3.1 $)^{55}$. They describe the diffractive spreading that occurs after point excitation of a grating. An example of one of these functions in operation is given in Figure 18, where a grating with a slanted input boundary is excited with a narrow focused beam; the spreading out over a triangular region bounded by the upper and lower Bragg angles is plain. Notice the fine periodic pattern in the photograph; this is caused by the interference of Bloch waves ${ }^{57}$. Since both branches of the stop-band are excited simultaneously in Figure 18, sharp interference fringes form between Bloch waves on opposite sides. These fringes have a spatial period $\Lambda_{f}=2 \pi /|\delta|$, where $\delta$ is the wavevector drawn between two points in reciprocal space associated with spatially coincident Bloch waves. The fringe orientation is normal to $\delta$. For a weak grating, the position of these fringes in space is accurately described by the zeros in the Bessel functions in Appendix A3.1. An intriguing situation arises when the group velocities of both Bloch waves point across the fringes; this appears to violate power conservation. In fact, no visible fringes (in the form of spatial variations in power density) form in the total field under these circumstances; instead, a kind of virtual interference somewhat akin to the "interference" of orthogonally polarized waves takes place ${ }^{57}$. In the photographs, the fringe visibility was enhanced by using a polarizer to block out the scattering from the upward or downward constituent plane waves of the fields.

In strongly modulated structures, interference may be suppressed owing to the disappearance of the left hand stop-band branch; as we have already seen, the wavevector diagram then becomes quite distorted (Figure 14), with "photonic band window" regions (where the group velocity hardly changes direction over an entire half Brillouin zone, pointing only very slightly normal to the planes). This is the wavevector diagram's view of resonant tunnelling across a waveguide array. Under the tight-binding approximation, the Wannier function is a spot the size of a unit cell, i.e., approximately a waveguide mode. Once excited, this waveguide mode spreads out sideways into the adjacent guides (see Figure 14 and Appendix A3.2).

A simple example of propagation in a spatially non-uniform structure, illustrating use of the effective mass method, is given in Figures 19a\&b. A region of periodic waveguide, with straight parallel boundaries and a slowly changing average index that peaks in the centre, is excited by a narrow focused beam (see figure caption for more details).

Note in conclusion that it is not possible to set up a simple Newtonian model for cases where the grating period and strength vary with position, because under these circumstances the curvature of the $H_{o}-\mathbf{k}$ diagram (and hence the effective mass tensor) depends both on position and the previous history of the ray path. In this case, a split-step propagation method can be used, finite steps through real and reciprocal space being made alternately, matching ray direction and phase velocity in each cycle.

\section{EXTENSION TO MULTIPLY PERIODIC STRUCTURES}

There has already been a focused and successful effort in Europe and North America on calculating the photonic band structure of multiply periodic PBG structures in two and three dimensions ${ }^{1,7,9-22}$. This work is covered in other chapters within this volume. But how useful is the intuitive picture developed in this chapter for understanding the 
behaviour of these more complicated media? The various tools we have introduced (Brillouin diagrams, wavevector diagrams, band edge diagrams) remain indispensable if all aspects of propagation are to be well understood. The tight-binding and nearly free approximations are also still valid, although the nature of the micro-resonances is
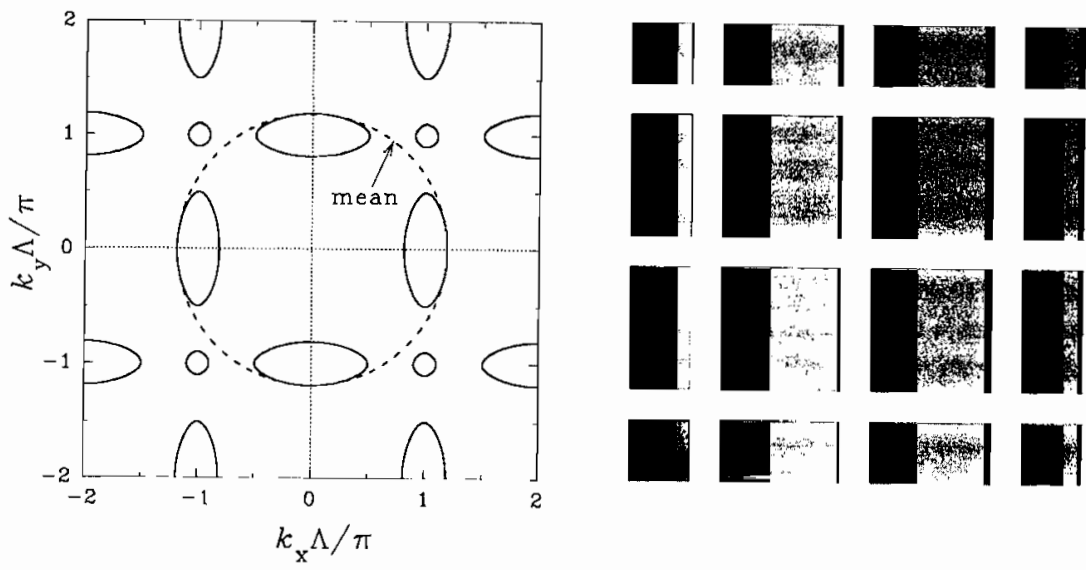

Figure 20. Wavevector diagram (left) for cubic lattice depicted on the right. The diagram was calculated using the method in references $16 \& 22$ for $n_{R}=0.47, \tau=a_{2} / a_{1}=0.56, \nu=1.19 \pi, n_{a v}$ $=\sqrt{ }\left\{\left(a_{1} n_{1}{ }^{2}+a_{2} n_{2}{ }^{2}\right) / A\right\}=1.8$ where $a_{1}$ and $a_{2}$ are the areas of the high and low index regions within a unit cell, whose area is $A$. For example, $400 \mathrm{~nm}$ wide square pillars of $\mathrm{Ta}_{2} \mathrm{O}_{5}$, separated by $200 \mathrm{~nm}$ air gaps would give this wavevector diagram at a wavelength of $1515 \mathrm{~nm}$ in vacuo.

considerably more complicated in three dimensions. Also, the second term in (11) can no longer be ignored - coupling between orthogonal field components occurs at the interfaces in the unit cells, resulting in polarization mixing. The tight-binding approximation can be usefully generalized to three dimensions, whether the picture is of coupled arrays of tubular waveguides, dielectric boxes, ellipsoids, spheres or more complicated entities. All these will support micro-resonances if excited appropriately (i.e., correct wavevector and frequency), in which case an analysis based on nearest-neighbour coupling can be used (see section 1.2.6).

Compared to the electronic case (with its simpler scalar wave equation), the calculation of photonic band structure is actually easier in one respect: the potential in each unit cell is precisely known, and does not change as the photons are redistributed (in the absence of optical nonlinearities). This means that band structure calculations for photons are actually more accurate.

The success of structures in which low index islands are surrounded by narrow seas of high index material (as in Yablonovitch's crystals ${ }^{7}$ ) may be due to the flattening of the Brillouin zone boundary owing to the absence of high- $Q$ micro-resonances that would tend to pull (when resonant) the band gap edges together, at the same time forcing the band gap to lower or higher values.

Finally, the wavevector diagram generalises very nicely to multiply periodic structures in two dimensions, and an example of how it looks for a cubic lattice (calculated using the method reported by Pendry and MacKinnon ${ }^{16,22}$ ) is given in Figure 20. Some more examples are available, together with two-dimensional field intensity profiles, in 
reference 5. A useful paper containing experimental results on propagation in doubly periodic planar waveguides has been written by Zengerle $^{58}$.

\section{MISCELLANEOUS TOPICS}

In this section we briefly discuss the relevance of our results on singly-periodic structures to a variety of different circumstances, including the properties and control of surface states and defect modes, the effects on photonic band structure of optical nonlinearity and optical gain, the extension to multiply periodic structures, and applications of photonic band gap materials.

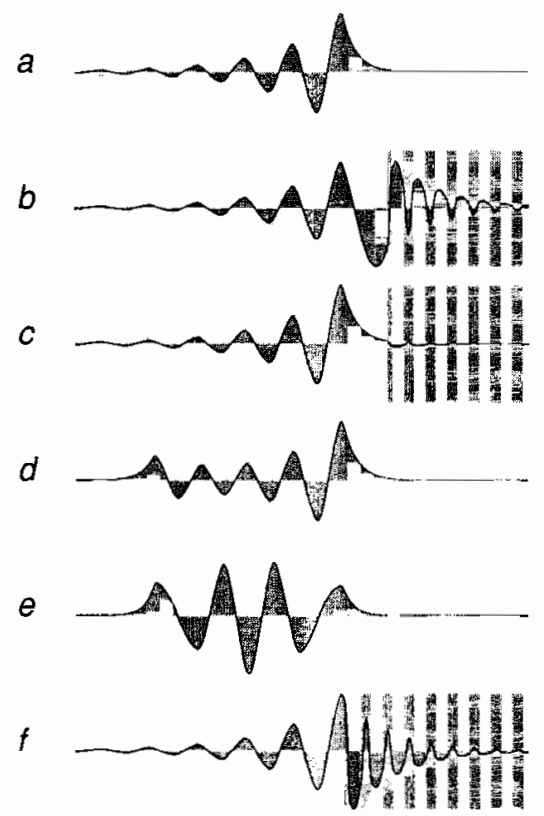

Figure 21. Different types of defect modes that can exist on or between multi-layer stacks: a) single surface guided mode (SGM); b) travelling waves bounded by Bragg reflection (high index intervening layer); c) two SGM's coupled by evanescent fields (low index intervening layer); d) two coupled SGM's on opposite sides of same stack; e) bound mode of finite stack formed from two travelling Bloch waves; f) SGM at the interface between two different multi-layer stacks.

\subsection{Localised Modes, Defects and Surface-Guided States}

As with any wave, Bloch waves can be quantized by truncating the medium in which they exist. This has the effect of reducing the spectral density of states (i.e., the number of states per unit frequency in a given volume of material) by localising them between the boundaries. Several other different sorts of localised modes can exist in multi- 
layer stacks, however, as illustrated in Figure 21 ; most (not all) of these have been previously reported in the literature ${ }^{75-87}$. The range of possibilities is much richer than in isotropic media, mainly due to one thing: total reflection can be produced in ranges of $\beta$ (the band gaps) where, based on $n_{a v}$, the stack would be expected to be transparent. This
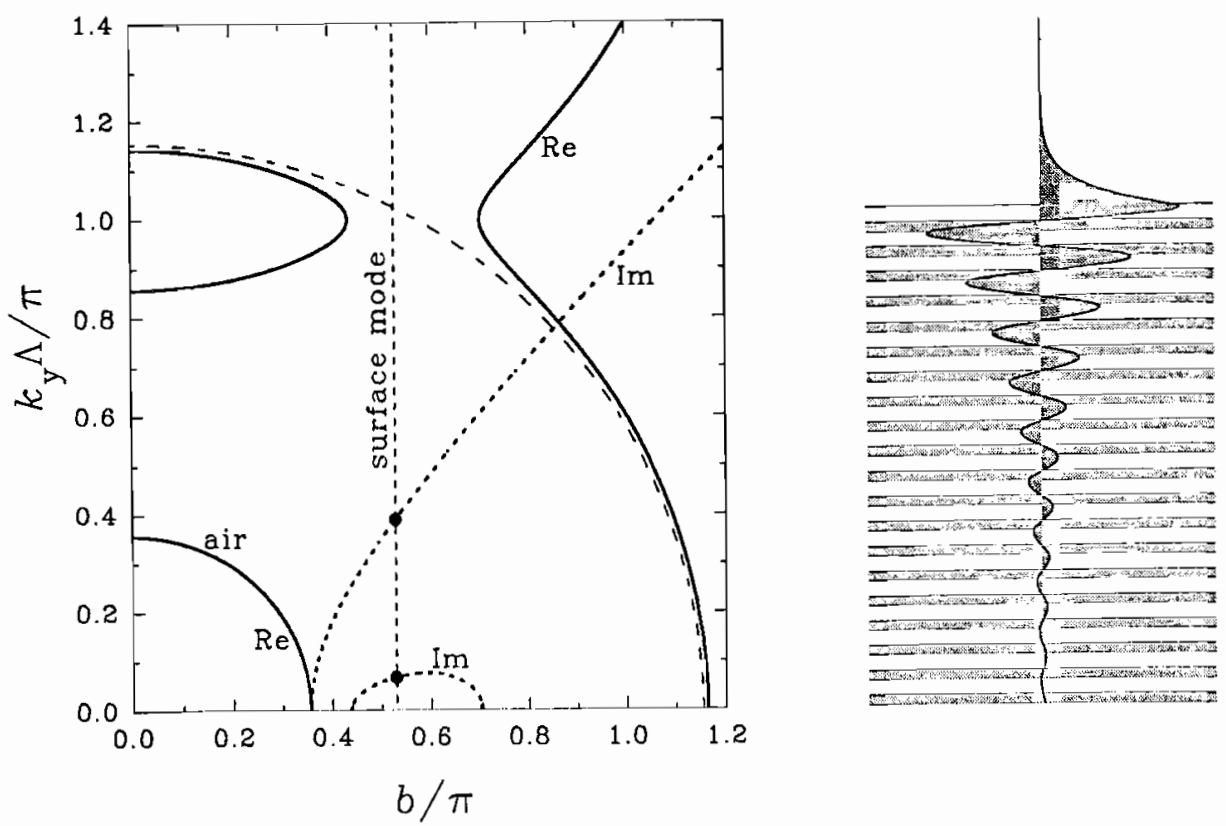

Figure 22. Wavevector diagram illustrating condition for formation of a particular surface guided mode ( $\tau=0.59, n_{R}=0.84, \nu=1.15 \pi$; last TE polarised; amplitude distribution depicted on the right). The parameters are for an AlAs/GaAlAs stack at $830 \mathrm{~nm}$, and the thickness of the final high index layer, normalised to $\Lambda$, is 0.036 . As the thickness of this layer is reduced, the $\beta$ value of the mode moves from the right-hand side of the stop-band to the left.

means that a single interface mode in each polarisation state can exist between the surface of a stack and a medium of low refractive index. The general approach to finding the bound modes is straightforward provided the expressions for the field distributions are chosen carefully, with the field distribution in the defect layer between two stacks referred to the centre as in (20). For a single surface mode the analysis is particularly simple, leading to the following dispersion equation:

$$
p_{j}\left(y_{e}-y_{j}\right)-\arctan \left[\frac{\xi_{e} p_{e} \Lambda a_{j}+b_{j}}{\xi_{j} p_{j} \Lambda a_{j}-\left(\xi_{e} p_{e} / \xi_{j} p_{j}\right) b_{j}}\right]=m \pi
$$

where the parameters with subscripts $j$ are those belonging to the last partial layer of the stack, the position of the edge is $y=y_{e}$ and the amplitude decay rate into the external isotropic medium is:

$$
p_{e}=\sqrt{\beta^{2}}-\overline{\left(k n_{e}\right)^{2}}
$$

where $n_{e}$ is the external index and $\xi_{e}=1$ for TE and $1 / n_{e}{ }^{2}$ for TM polarisation. The conditions for formation of a surface mode are best understood by reference to the wavevector diagram (Figure 22), because this reveals clearly the location of the band gaps 
for a particular stack, and hence the ranges of $\beta$ within which a surface wave can exist. They may form for values of $\beta>k n_{e}$ that at the same time lie within a band gap of the stack, and they only exist if the final layer has an appropriate thickness. This means that they may easily be suppressed if desired (although perhaps not in both polarization states) by appropriately designing the stack. We plan to discuss these modes at length elsewhere. It is interesting to point out in addition that TE and TM surface plasmon modes with hugely different propagation constants can also exist at the interface between a metal and a stack; they may of course exist (since a metal forbids any propagation whatsoever) at normal incidence $\beta=0$, creating a resonator with just one mode. Similar stationary localised modes can also exist at the interface between two different multi-layer stacks. Surface photonic states are directly analogous to the electronic surface states in the solidstate, and can appear on multiple quantum well structures ${ }^{83}$.

\subsection{Effects of Optical Gain: Lasers}

Since one of the major applications of photonic band gap materials is in lasers, it is worthwhile considering the effect that optical gain may have on the passive dispersion. It has been known for a long time that the $\omega-k$ relationship is strongly affected if the gain $g$ (per unit length) and the grating strength $k$ (the well-known coupling constant defined in Appendix A3.1) are comparable, i.e., if:

$$
\kappa / g \sim 1
$$

or smaller; this is commonly the case in a distributed feed-back (DFB) semiconductor $\operatorname{laser}^{35,37}$. Alterations in $k_{y}$ for a given frequency $\omega$ (Figure 23) will of course alter the frequencies at which a cavity will resonate, which might be of importance in some contexts such as dense wavelength-division-multiplexing applications. In contrast to DFB lasers, this is unlikely to be a matter of much concern in PBG materials where the grating strength (perhaps $6 \pi$ per $\mu \mathrm{m}$ in Yablonovitch's proposed PBG AlGaAs structures ${ }^{7}$ ) is certain to be much greater than the gain. Of more concern is unwanted lasing outside the band gap. DFB lasers in their purest form consist of a defect-free length of grating - a kind of onedimensional PBG structure. Laser oscillation occurs at two frequencies symmetrically placed on either side of the band gap, where the photonic states are real while the reflection is still strong enough to provide substantial feedback. In materials with broad unwanted bands of high gain it may be difficult to produce a band gap wide enough to suppress lasing completely. The etching process used to produce a PBG in a semiconductor laser material will inevitably concentrate the gain in the high index regions. This will tend to favour Bloch modes whose photonic probability distribution peaks in the high gain regions, and may often result in suppression of laser oscillation on one side of the band gap. In the language of quantum photodynamics, the expectation value of the gain will be higher on one side of the band gap compared to the other. An early example of a related phenomenon is the Borrmann effect (1941), where x-rays experience anomalously low absorption at the Bragg condition in an $\alpha$-quartz crystal. This is caused by the $\mathrm{x}$-ray Bloch waves on the inner stop-band branch (see Figure 11) experiencing less absorption than those on the outer branch, since their probability distributions peak in between the atoms where the absorption is concentrated ${ }^{31}$.

\subsection{Effects of Optical Nonlinearity: Gap Solitons}

The ability to pack as many photons as desired into each photonic state means that optical Kerr nonlinearities can become important at high optical intensities. If the nonlinear index change is comparable to the index modulation depth in the PBG material, the stop- 


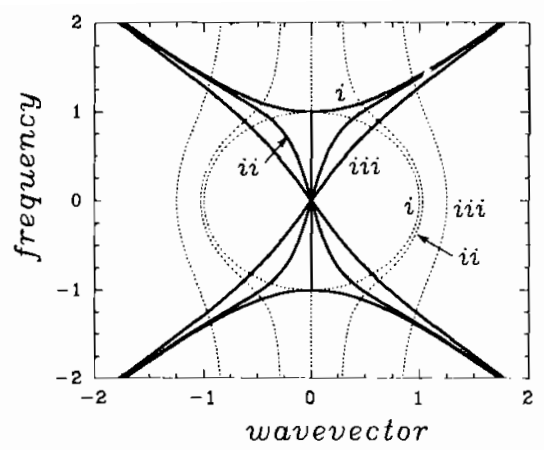

Figure 23. Stop band distortion in the presence of optical gain; the ratio of $g / \kappa$ is $0,0.25$ and 0.75 respectively in the three cases $i, i i$ and $i i i$. The vertical axis is frequency deviation from the Bragg condition, normalised to half the band gap width, and the horizontal axis is $k_{y}-K / 2$, normalised to the coupling constant $k$. The full and dotted lines are based on the real and imaginary parts of $k_{\mathrm{y}}$.

band branches become significantly distorted ${ }^{74}$; the dispersion and the nonlinearity are then closely entangled. As recently studied by John and Aközbek ${ }^{67}$, this can result in the appearance, within a linear three-dimensional photonic band gap, of real states induced by the nonlinearity. These densely occupied photonic states trapped by their own nonlinearity are in fact related to gap solitons ${ }^{68-73}$. The presence of optical nonlinearity outside the band gap in a region of strong spatial dispersion can also result in strong nonlinear beamsteering, since the group velocity of the Bloch waves is then a function of power.

We have seen that the effective mass of photons becomes small close to a band edge; this means that scattering centres that would have a negligible effect on free photons can strongly scatter a photon in a Bloch state. The interaction (collision cross-section) is greatly increased by the small group velocity (momentum) of the Bloch wave (photon). This has important implications in stimulated Brillouin scattering. Two travelling counterpropagating Bloch waves with frequencies close to the band edge on the same side of a band gap will be coupled together very strongly by a low frequency, low momentum acoustic wave $\mathrm{e}^{32,33}$. This is because the expectation value of the momentum difference between backward and forward Bloch waves can itself be very small, and the coupling strength depends on the reciprocal of the group velocity, which also goes to zero at the band edge. Raman scattering may similarly be enhanced if the band gap is sufficiently large to allow both the Stokes and anti-Stokes Bloch waves to possess small group velocities close to the same band edge. Nonlinear interactions across a band gap are unlikely to be significant since the Bloch functions do not overlap strongly spatially.

\subsection{Applications of Photonic Band Gap Materials}

Since PBG materials are essentially synthetic (although natural biology or bioengineering may be able to contribute some useful structures), there has to be a good reason for going to the trouble (and expense) of making them. Hence the importance of the question: What use are they? Interpreted in the widest sense, they may be viewed as synthetic effective index media with highly unusual anisotropic refractive indices, diffractive properties and substantial ranges of angle and frequency where propagation is 
forbidden. Independent control of phase and group velocity is possible, which is like saying that conventional optics (with only a restricted range of anisotropic molecular polarizabilities at its disposal - those naturally occurring in crystals) can be augmented or even superseded, with many new features.

A common problem in waveguide optics is creating phase-matched single modes in low and high index materials. In conventional guided wave optics, the mode indices
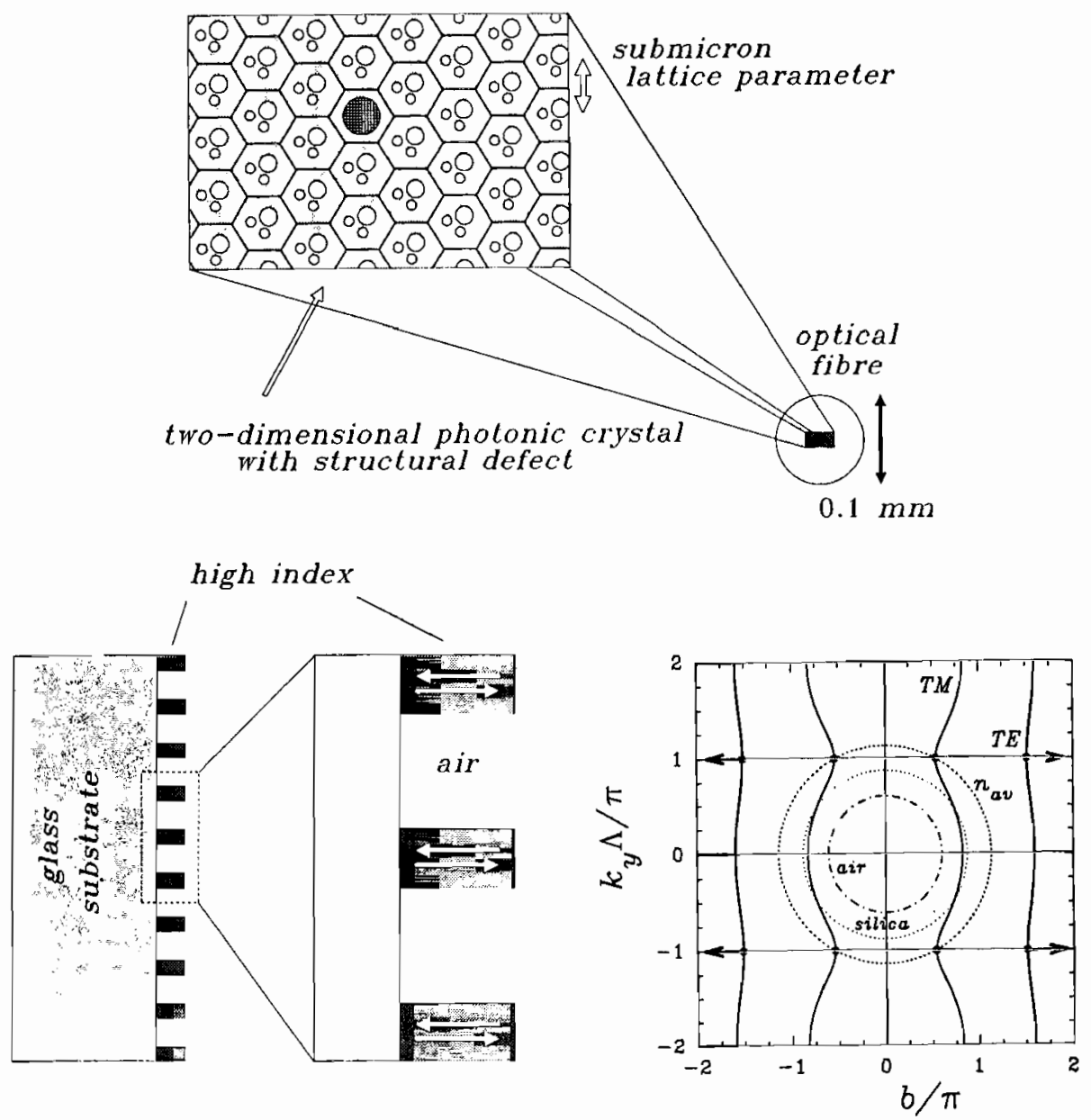

Figure 24. Two examples of structures that will exhibit unusual photonic band gap effects: (a) A holey fibre, whose core is a two-dimensional photonic crystal with structural defects where the light is trapped; strong anisotropy can be built in; and (b) A resonator made from a single periodically etched-through layer of dielectric material $\left(\mathrm{Ta}_{2} \mathrm{O}_{5}\right.$, index 2.12$)$ that completely confines light in a localised state. Its wavevector diagram is also shown; the group velocity at the Brillouin zone edge points normal to the layer, and the related Bloch wave cannot escape because its lowest order plane waves are totally internally reflected at the left and right boundaries.

must lie in between the substrate and the film index. Surface guided Bloch waves neatly side-step this restriction ${ }^{87}$, permitting for example a single surface mode on a high index multi-layer stack to be phase-matched to the light guided in a single-mode silica fibre, or enhancing the penetration of light into a low index external medium such as an aqueous solution containing some chemical to be sensed. This may have important potential 
applications in biological and chemical sensing. Multi-layer stacks in AlGaAs, for example, can be designed to support surface Bloch modes with indices lying between air and the maximum index of the stack (around 3.5).

There are many potential uses of localised single states in PBG materials ${ }^{77}$. These can be created by introducing a structural defect at one point in a grating; this could be a slight local variation in average index or grating modulation depth. When appropriately designed, a single-mode photonic defect state appears that occupies a volume of space substantially larger than is possible in a conventional single-mode two-mirror lasing cavity. Single-frequency laser oscillation then becomes feasible in materials whose optical gain is normally too low.

The creation of a structural defect, by the addition of less/more high index material in a unit cell, results in localised modes whose frequency lies within the band gap close to the upper/lower band edge; these may be regarded as donor/acceptor states by analogy with semiconductors ${ }^{76}$. The process of donation and acceptance involves shifting a photon between a localised and an almost-localised state (just outside the band gap) at a different - frequency. How this might be achieved is an interesting question - perhaps by the photonic crystal equivalent of an optical phonon?

Many interesting applications of PBG materials have already been reported ${ }^{4,5,9,17,39,40}$. Two examples from our own work of exotic structures with potentially useful properties are illustrated in Figure 24. The "holey" fibre (Figure 24a) is a glass optical fibre with a two-dimensional crystalline core region and a central defect that traps photons while permitting them to travel along the fibre axis. Many useful properties can be built into the crystal structure, such as various types of optical anisotropy, and very strong lateral confinement can be achieved in the photonic band window range of the band edge diagram (Figure 13). An unusual resonator forms if a high index film is etched with a periodic pattern as illustrated in Figure $24 \mathrm{~b}$. At the correct wavelength and film thickness, a tightly confined stationary optical mode exists, with zero group velocity along the film. This cavity, made entirely from a small volume of material, could have a very high $Q$ and be phase-matched to an optical fibre mode.

In one important respect, band gap engineering - the optimisation of the performance of a PBG crystal by changing the geometry of the unit cell - may be somewhat easier for photons, at least at the design stage. This is because the "grainy-ness" of dielectric material is much less for photons than for electrons (atoms are not easily divisible!). Hence it is likely that new effects can be "engineered in" based on features fractions of a period in thickness; for example, anti-surface wave layers could be coated on to a multi-layer stack or a PBG crystal to prevent lasing in unwanted surface modes.

\section{IN CONCLUSION}

In moving away from a traditional plane wave approach towards one based on Bloch waves, photonic band theory provides an alternative conceptual framework for thinking about light in periodic structures, and provides the starting point for a full "quantum photo-dynamical" description of photons in PBG media. This is well illustrated by the discussion of electromagnetic wave propagation in singly-periodic structures presented here. For many years, the response of laser designers to a problem of lifetimes and unwanted transitions has been to try to alter the electronic properties of the material itself. This has paid off handsomely in the quaternary III-V system, and seems set to do so again in the II-VI system for blue lasers ${ }^{42}$. Photonic band gap materials offer an additional powerful means of altering the fluorescence properties of a material without having to tamper directly with its electronic structure. The implications of a Bloch wave approach, brought into the limelight by Yablonovitch's proposal that photonic band gaps 
could exist by analogy with electronic band gaps, have still to be fully worked out. One immediate benefit, however, is a sea-change in approach within optics that seems certain to lead to new applications based on a re-interpretation of the physics of light in periodic structures.

\section{ACKNOWLEDGEMENTS}

The authors wish to thank Jacek Generowicz for carrying out the numerical modelling for Figure 20, Tim Sluckin and Chris Pannell for many useful discussions, and Dale Atkin for checking the manuscript. Partial support for the work on surface guided modes is provided by the U.S. Department of the Air Force through the European Office of Aerospace Research and Development, London, and the PBG fibre work is being supported by the Defence Research Agency, Malvern. The experimental results were obtained by Philip Russell at the Technische Universität Hamburg-Harburg in 1982-84, with the support of the Alexander von Humboldt Foundation. The Optoelectronics Research Centre is funded by the U.K. Science and Engineering Research Council.

\section{REFERENCES AND READING GUIDE}

The references are sorted into subjects, so do not appear in numerical order in the text. The asterisked items are suggested sources for introductory reading or background material.

\section{Photonic Band Gaps}

1.* W. van Haeringen and D. Lenstra (Editors), "Analogies in Optics and Microelectronics," Kluwer Academic Publishers, Dordrecht (1990).

2.* $\quad$ S. John, "Localization of light," Physics Today 44 (32-40) 1991

3.* I. Amato, "Designing crystals that say no to photons," Science, 255 (1512) 1992.

4.* H.O. Everitt, "Applications of photonic band gap structures," Optics and Photonics News (2023), November 1992.

5.* P.St.J. Russell, "Photonic Band Gaps," Physics World 5 (37-42) 1992.

6.* C.M. Bowden, J.P. Dowling and H.O. Everitt (Editors), "Development and Applications of Materials Exhibiting Photonic Band Gaps," feature section in J. Opt. Soc. Am. 10 (279-413) 1993.

7.* $\quad$ E. Yablonovitch, "Photonic band gap structures," J. Opt. Soc. Am. 10 (283-295) 1993

8.* Special issue of $J$. Modern Optics on Photonic band structure, to be published, 1994.

9. G. Kurizki and A.Z. Genack, "Suppression of molecular interactions in periodic dielectric structures," Phys. Rev. Lett. 61 (2269-2271) 1988.

10. K.M. Ho, C.T. Chan and C.M. Soukoulis, "Existence of a photonic band gap in periodic dielectric structures," Phys. Rev. Lett. 65 (3152-3155) 1990.

11. K.M. Leung and Y.F. Liu, "Full vector wave calculation of photonic band structures in face-centered-cubic dielectric media," Phys. Rev. Lett. 65 (2646-2649) 1990.

12. Z. Zhang and S. Satpathy, "Electromagnetic wave propagation in periodic structures: Bloch wave solution of Maxwell's equations," Phys. Rev. Lett. 65 2650-2653) 1990.

13. M. Plihal and A.A. Maradudin, "Photonic band structure in two-dimensional systems: The triangular lattice," Phys. Rev. B44 (8565-8571) 1991.

14. E. Yablonovitch and T.J. Gmitter, "Photonic band structure: The face-centered-cubic case employing nonspherical atoms," Phys. Rev. Lett. 67 (2295-2298) 1991.

15. P. R. Villeneuve and M. Piché, "Photonic band gaps in two-dimensional square lattices: Square and circular rods," Phys. Rev. B46 (4973-4975) 1992.

16. J.B. Pendry and A. MacKinnon, "Calculation of photon dispersion relation," Phys. Rev. Lett., $69(2772-2775) 1992$.

17. E.R. Brown, C.D. Parker and E. Yablonovitch, "Radiation properties of a planar antenna on a 
photonic-crystal substrate," J. Opt. Soc. Am. 10 (404-407) 1993.

18. S.Y. Lin and G. Arjavalingam, "Tunnelling of electromagnetic waves in two-dimensional photonic crystals," Opt. Lett. 18 (1666-1668) 1993.

19. A.A. Maradudin and A.R. McGurn, "Photonic band structure of a truncated, two-dimensional, periodic dielectric medium," J. Opt. Soc. Am. 10 (307-313) 1993.

20. R.D. Meade, A.M. Rappe, K.D. Brommer and J.D. Joannopolous, "Nature of the photonic band gap: some insights from a field analysis," J. Opt. Soc. Am., 10 (328-332) 1993.

21. H. S. Sözüer and J.W. Haus, "Photonic bands: simple-cubic lattice," J. Opt. Soc. Am. 10 (296302) 1993.

22. J.B. Pendry, "Photonic band structures," to be published in J. Mod. Opt., 1994.

\section{Electrons in Solids}

23.* N.W. Ashcroft and N.D. Mermin, "Solid State Physics," Harcourt Brace College Publishers, Fort Worth (1976).

24.* J.S. Blakemore, "Solid State Physics," Cambridge University Press, Cambridge, 1991.

25.* H. Haken, "Quantum Field Theory of Solids," North-Holland, Amsterdam (1988).

25. C. Kittel, "Quantum Theory of Solids," John Wiley \& Sons, New York (1987).

\section{Electromagnetic Waves and Periodic Structures}

26.* J.R. Reitz, F.J. Milford and R.W. Christy, "Foundations of Electromagnetic Theory," 3rd edition, Addison-Wesley Publishing Company,Massachusetts (1979).

27.* L. Solymar and D.J. Cooke, "Volume Holography and Volume Gratings," Academic Press, London (1981).

28.* P. Yeh, A. Yariv and C.-S. Hong, "Electromagnetic propagation in periodic stratified media. I \& II," J. Opt. Soc. Am. 67 (423-448) 1977

29.* P. Yeh, "Optical Waves in Layered Media," Wiley, New York (1988).

30. L. Brillouin, "Wave Propagation in Periodic Structures," McGraw-Hill, New York (1946); "Wave Propagation and Group Velocity," Academic Press, New York (1960).

31. P.St.J. Russell and L. Solymar, "Borrmann-like anomalous effects in volume holography," Appl. Phys. 22 (335-353) 1980.

32. P.St.J. Russell, "Bragg resonance of light in optical superlattices," Phys. Rev. Lett. 56 (596-599) 1986.

33. P.St.J. Russell, "Optical superlattices for modulation and deflection of light," J. Appl. Phys. 59 (3344-3355) 1986.

34. E.N. Economou and A. Zdetsis, "Classical wave propagation in periodic structures," Phys. Rev. B40 (1334-1337) 1989.

\section{Distributed Feedback Lasers and Micro-Cavities}

35.* H. Kogelnik and C.V. Shank, "Coupled-wave theory of distributed feedback lasers," J. Appl. Phys. 43 (2327-2335) 1972.

36.* D.S. Chemla (editor), "Optics of Nanostructures," special issue of Physics Today 46 (22-73) 1993.

37.* F.K. Kneubühl, "Theories of Distributed Feedback Lasers," Harwood Academic Publishers, Switzerland (1993).

38. S.L. McCall and P.M. Platzman, "An optimised $\pi / 2$ distributed feedback laser," IEEE J. Quant. Electr. QE-21 (1899-1904) 1985.

39. D.L. Bullock, C.-C. Shih and R.S. Margulies, "Photonic band structure investigation of twodimensional Bragg reflector mirrors for semiconductor laser mode control," J. Opt. Soc. Am. 10 (399-403) 1993.

40. T. Erdogen, K.G. Sullivan and D.G. Hall, "Enhancement and inhibition of radiation in cylindrically symmetric periodic structures," J. Opt. Soc. Am. 10 (391-398) 1993

41. Y. Yamamoto and R.E. Slusher, "Optical processes in microcavities," Physics Today 46 (66-73) 1993.

42. N. Nakayama, S. Itoh, T. Ohata, K. Nakano, H. Okuyama, M. Ozawa, A. Ishibashi, M. Ikeda and Y. Mori, "Roon temperature continuous operation of blue-green laser diodes, "Electr. Lett. 29 (1488-1489) 1993. 


\section{Optics, Waveguides and Optoelectronics}

43.* E. Hecht and A. Zajac, "Optics," Addison-Wesley Publishing Company, Massachusetts (1974).

44.* M. Born and E. Wolf, "Principles of Optics," fifth edition, Pergamon Press, Oxford (1975).

45.* T. Tamir (editor), "Integrated Optics," Springer-Verlag, Berlin (1979).

46.* H. Haken, "Light," volume 1: Waves, Photons, Atoms, North Holland, Amsterdam (1981).

47.* A. Korpel (Editor), "Selected Papers on Acoustooptics," SPIE Optical Engineering Press, volume MS16, Bellingham, Washington (1990).

48." D. Marcuse, "Theory of Dielectric Optical Waveguides," 2nd edition, Academic Press, Boston (1991).

49.* A. Yariv, "Optical Electronics," 4th edition, Harcourt Brace College Publishers, Fort Worth, (1991).

50.* H.A. Haus, "Waves and Fields in Optoelectronics," Prentice-Hall, Englewood Cliffs (1984).

51. J.A. Arnaud, "Beam and Fibre Optics," Academic Press, New York (1976).

52. E. Garmire, Semiconductor components for monolithic applications, in: "Integrated Optics, ${ }^{\text {T }}$ T. Tamir, ed., Springer-Verlag, Berlin (1979).

53. A.W. Snyder and J.D. Love, "Optical Waveguide Theory," Chapman and Hall, London (1983).

54. P.St.J. Russell and R. Ulrich, "Elementary and coupled waves in periodic planar waveguides," 2nd European Conference on Integrated Optics, Florence, IEE Conf. Publication 227 (88-91) October 1983.

55. P.St.J. Russell, "Novel thick-grating beam-squeezing device in $\mathrm{Ta}_{2} \mathrm{O}_{5}$ corrugated planar waveguide," Electr. Lett. 20 (72-73) 1984.

56. P.St.J. Russell, "Optics of Floquet-Bloch waves in dielectric gratings," Appl. Phys. B39 (231-246) 1986.

57. P.St.J. Russell, "Interference of integrated Floquet-Bloch waves," Phys. Rev. A 33 (3232-3242) 1986.

58. R. Zengerle, "Light propagation in single and doubly periodic planar waveguides," J. Mod. Opt. 34 (1589-1617) 1987.

\section{X-ray Diffraction}

59.* J.M. Bijvoet, W.G. Burgers and G. Hägg, "Early Papers on Diffraction of X-rays by Crystals," A. Oosthoek's Uitgeversmaatschappil N.V., Utrecht (1969).

60.* P.P. Ewald, "Fifty Years of X-ray Diffraction," A. Oosthoek's Uitgeversmaatschappil N.V., Utrecht (1962).

61.* Z.G. Pinsker, "Dynamical Scattering of X-rays in Crystals," Springer-Verlag, Berlin (1978).

62.* J.M. Cowley, "Diffraction Physics," second revised edition, North-Holland (1981).

63. C.G. Darwin, "The theory of x-ray reflexion," Phil. Mag. 27 (315-) 1914.

\section{Nonlinear Optics}

64.* Y.R. Shen, "Principles of Nonlinear Optics," Wiley Interscience, New York (1984).

65.* A.D. Boardman, M. Bertolotti and T. Twardowski (editors), "Nonlinear Waves in Solid State Physics," NATO ASI Series: Series B: Physics, volume 247, Plenum, New York (1993).

66.* P.N. Butcher and D. Cotter, "The Elements of Nonlinear Optics," Cambridge Studies in Modern Optics, volume 9, Cambridge University Press, Cambridge (1993).

67.* S. John and N. Aközbek, "Nonlinear optical solitary waves in a photonic band gap," Phys. Rev. Lett. 71 (1168-1171) 1993.

68. D.L. Mills and S.E. Trullinger, "Gap solitons in nonlinear periodic structures," Phys. Rev. B 36 (947-952) 1987.

69. W. Chen and D.L. Mills, "Gap solitons and the nonlinear optical response of superlattices," Phys. Rev. Lett. 58 (160-164) 1987.

70. J.E. Sipe and H.G. Winful, "Nonlinear Schrödinger solitons in a periodic structure," Opt. Lett. 13 (133-134) 1988.

71. C.M. de Sterke and J.E. Sipe, "Envelope-function approach for the electrodynamics of nonlinear periodic structures," Phys. Rev. A38 (5149-5165) 1988.

72. A.B. Aceves and S. Wabnitz, "Self-induced transparency solitons in nonlinear refractive periodic media," Phys. Lett. A141 (37-42) 1989.

73. D.N. Christodoulides and R.I. Joseph, "Slow Bragg solitons in nonlinear periodic structures," Phys. Rev. Lett. 62 (1746-1749) 1989. 
74. P.St.J. Russell and J.-L. Archambault, "Field microstructure and temporal and spatial instability of photonic Bloch waves in nonlinear periodic media," to be published in J. de Physique III, 1994; see also paper FrD4, International Quantum Electronics Conference, IQEC'92, Vienna, Austria, June 1992.

\section{Surface States and Defects}

75.* P. Yeh, "Optical Waves in Layered Media," Wiley, New York (1988).

76.* E. Yablonovitch, T.J. Gmitter, R.D. Meade, A.M. Rappe, K.D. Brommer and J.D. Joannopolous, "Donor and acceptor modes in photonic band structure," Phys. Rev. Lett. 67 (3380-3383) 1991.

77.* D.R. Smith, R. Dalichaouch, N. Kroll, S. Schultz, S.L. McCall and P.M. Platzman, "Photonic band structure and defects in one and two dimensions," J. Opt. Soc. Am. 10 (314-321) 1993.

78. P. Yeh, A. Yariv, A. Cho, "Optical surface waves in periodic layered media," Appl. Phys. Lett. 32 (104-105) 1978.

79. W. Ng, P. Yeh, P.C. Chen and A. Yariv, "Optical surface waves in periodic layered medium grown by liquid phase epitaxy," Appl. Phys. Lett. 32 (370-371) 1978.

80. A.A. Bulgakov and V.R. Kovtun, "Surface optical oscillations in a bounded layered periodic medium," Opt. Spectrosc. (USSR) 56 (47I-472) 1984.

81. A.A. Bulgakov and V.R. Kovtun, "Study of surface optical oscillations in periodic multi-layer media," Solid State Comm. 56 (781-785) 1985.

82. S.D. Gupta and B. Buti, "Electromagnetic surface waves in periodic layered media," Indian J. Pure \& Appl. Phys. 23 (452-457) 1985.

83. F. Huang, "Electronic surface states confined to the boundary of periodic multiple quantum wells," Appl. Phys. Lett. 57 (1669-1671) 1990.

84. R.D. Meade, K.D. Brommer, A.M. Rappe and J.D. Joannopoulos, "Electromagnetic Bloch waves at the surface of a photonic crystal," Phys. Rev. A 44 (10961-10964) 1991.

85. K.M. Leung, "Defect modes in photonic band structures: a Green's function approach using vector Wannier functions," J. Opt. Soc. Am. 10 (303-306) 1993.

86. R.P. Stanley, R. Houdré, U. Oesterle, M. Ilegems and C. Weisbuch, "Impurity modes in onedimensional systems: The transition from photonic band gaps to microcavities, "Phys. Rev. A 48 (2246-2249) 1993.

87. T.A. Birks, F.D. Lloyd-Lucas and P.St.J. Russell, "Applications of optical surface waves on multi-layer stacks," in preparation, 1994.

\section{APPENDIX A1. MAIN MATHEMATICAL SYMBOLS AND THEIR MEANINGS}

Roman characters

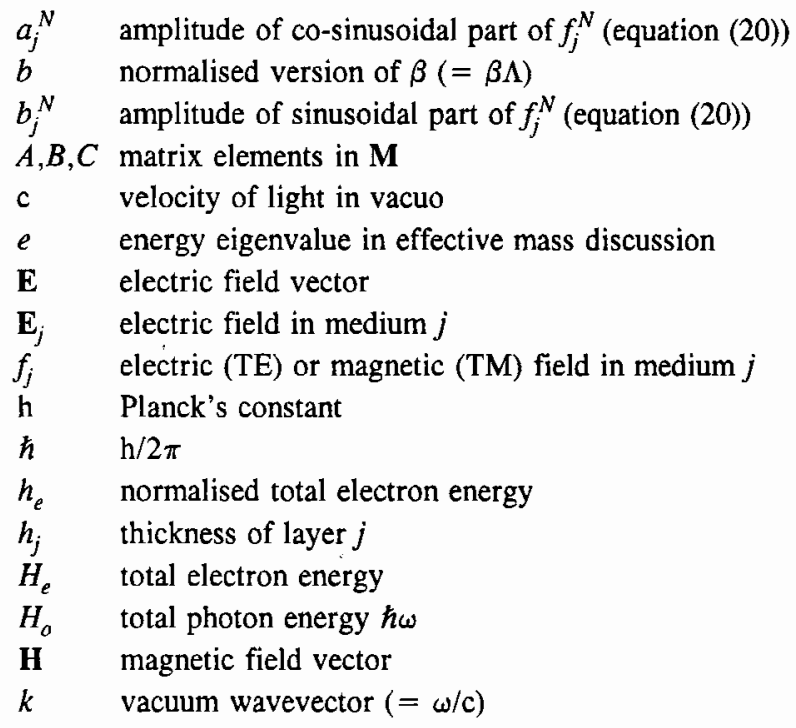




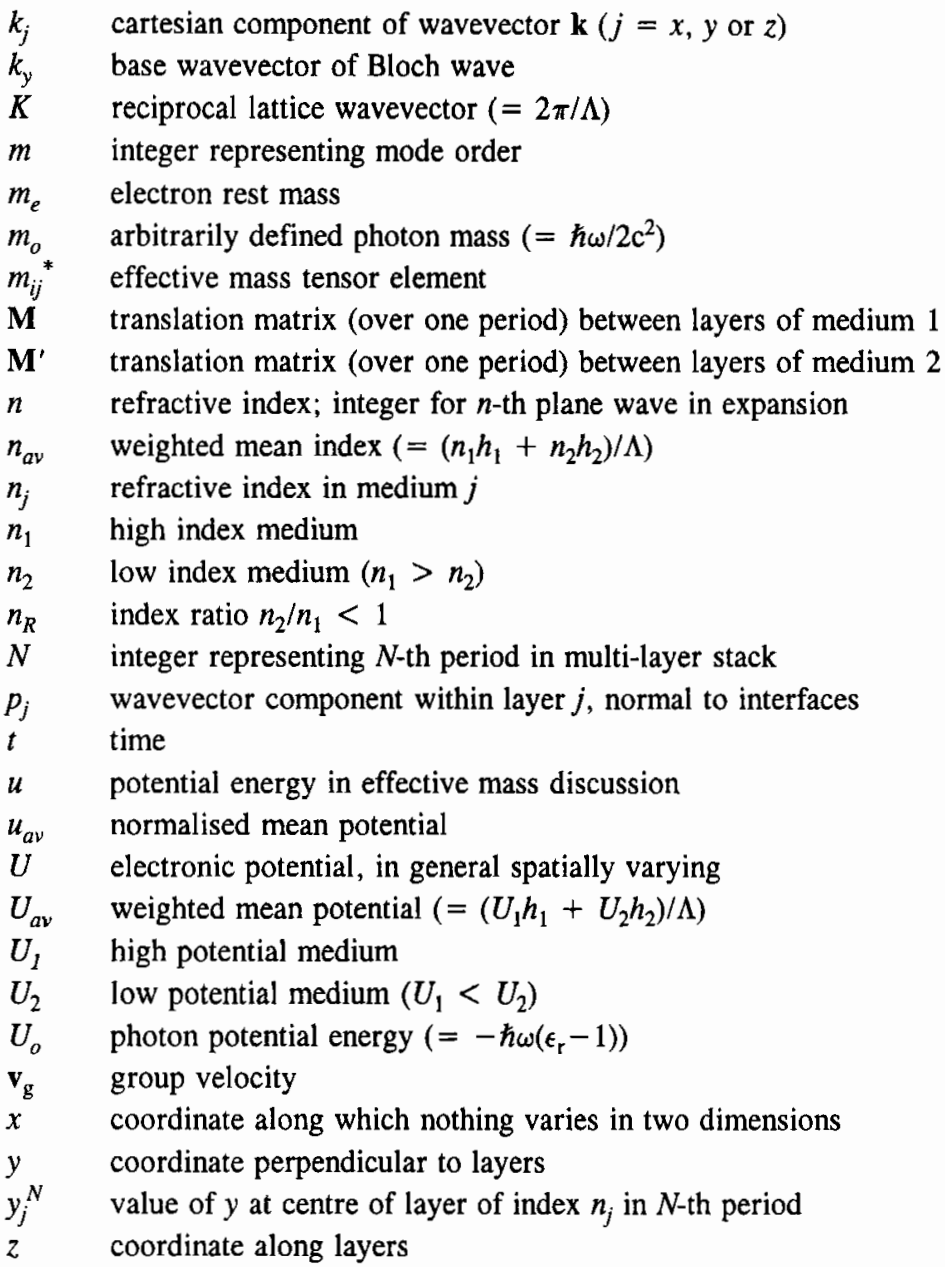

\section{Greek characters}

$\beta \quad$ wavevector component along the layers ( $z$ direction)

$\epsilon_{o} \quad$ dielectric permittivity of free space

$\epsilon_{\mathrm{r}} \quad$ relative dielectric constant

$\theta_{c} \quad$ critical angle for total internal reflection (between ray and normal to interface)

$\Lambda \quad$ period in multi-layer stack $\left(=h_{1}+h_{2}\right)$

$\mu_{\mathrm{o}} \quad$ magnetic permeability of free space

$\nu \quad$ normalised frequency $\left(=k n_{a v} \Lambda\right)$

$\xi_{j} \quad=1$ for TE polarised light

$=1 / n_{j}^{2}$ for TM polarised light

$\tau$, relative thickness of layer $2\left(=h_{2} / \Lambda\right)$

$\psi \quad$ general field amplitude

$\omega / 2 \pi$ optical frequency

\section{APPENDIX A2. TRANSLATION MATRIX ELEMENTS}

The matrix $\mathbf{M}_{21}$ relating the field in the 2 nd layer to the field in the 1st layer is 


$$
\left(\begin{array}{l}
a_{2}^{N} \\
b_{2}^{N}
\end{array}\right)=\mathbf{M}_{21}\left(\begin{array}{l}
a_{1}^{N} \\
b_{1}^{N}
\end{array}\right)=\left(\begin{array}{ll}
A_{21} & B_{21} \\
C_{21} & D_{21}
\end{array}\right)\left(\begin{array}{l}
a_{1}^{N} \\
b_{1}^{N}
\end{array}\right),
$$

where

$$
\begin{aligned}
A_{21} & =c_{1} c_{2}-\left(\xi_{1} p_{1} \Lambda / \xi_{2} p_{2} \Lambda\right) s_{1} s_{2}, \\
B_{21} & =s_{1} c_{2} /\left(\xi_{1} p_{1} \Lambda\right)+c_{1} s_{2} /\left(\xi_{2} p_{2} \Lambda\right), \\
C_{21} & =-\xi_{1} p_{1} \Lambda s_{1} c_{2}-\xi_{2} p_{2} \Lambda c_{1} s_{2}, \\
D_{21} & =c_{1} c_{2}-\left(\xi_{2} p_{2} \Lambda / \xi_{1} p_{1} \Lambda\right) s_{1} s_{2}, \\
& \operatorname{det} \mathbf{M}_{21}=1,
\end{aligned}
$$

where the terms $s_{j}$ and $c_{j}$ are shorthand for:

$$
c_{j}=\cos \left(p_{j} h_{j} / 2\right), \quad s_{j}=\sin \left(p_{j} h_{j} / 2\right) .
$$

The matrix $\mathbf{M}_{12}$ relating the field in the 1st layer of the $(N+1)$ th period to the field in the 2nd layer of the $N$ th period is then

$$
\left(\begin{array}{l}
a_{1}^{N+1} \\
b_{1}^{N+1}
\end{array}\right)=\mathbf{M}_{12}\left(\begin{array}{l}
a_{2}^{N} \\
b_{2}^{N}
\end{array}\right)=\left(\begin{array}{ll}
D_{21} & B_{21} \\
C_{21} & A_{21}
\end{array}\right)\left(\begin{array}{l}
a_{2}^{N} \\
b_{2}^{N}
\end{array}\right) .
$$

The analysis can either be based on the translation matrix $\mathbf{M}=\mathbf{M}_{12} \mathbf{M}_{21}$ (with a state vector representing the field in layers with index $n_{1}$ ) or equivalently on the matrix $\mathbf{M}^{\prime}=$ $\mathbf{M}_{21} \mathbf{M}_{12}$ (state vector representing the field in layers with index $n_{2}$ ). $\mathbf{M}$ is

$$
\left(\begin{array}{l}
a_{1}^{N+1} \\
b_{1}^{N+1}
\end{array}\right)=\mathbf{M}\left(\begin{array}{l}
a_{1}^{N} \\
b_{1}^{N}
\end{array}\right)=\left(\begin{array}{ll}
A & B \\
C & D
\end{array}\right)\left(\begin{array}{l}
a_{1}^{N} \\
b_{1}^{N}
\end{array}\right),
$$

where

$$
\begin{gathered}
A=D=A_{21} D_{21}+B_{21} C_{21}, \\
B=2 D_{21} B_{21}, \quad C=2 A_{21} C_{21} .
\end{gathered}
$$

$A$ can be re-arranged as

$$
A=\cos \left(p_{1} h_{1}\right) \cos \left(p_{2} h_{2}\right)-\frac{1}{2}\left(\frac{p_{1} \xi_{1}}{p_{2} \xi_{2}}+\frac{p_{2} \xi_{2}}{p_{1} \xi_{1}}\right) \sin \left(p_{1} h_{1}\right) \sin \left(p_{2} h_{2}\right)
$$

but $B$ and $C$ are most conveniently expressed as the product of two factors as above. The elements of the alternative matrix $\mathbf{M}^{\prime}$ are:

$$
A^{\prime}=D^{\prime}=A \text {, }
$$




$$
B^{\prime}=2 A_{21} B_{21}, \quad C^{\prime}=2 D_{21} C_{21} .
$$

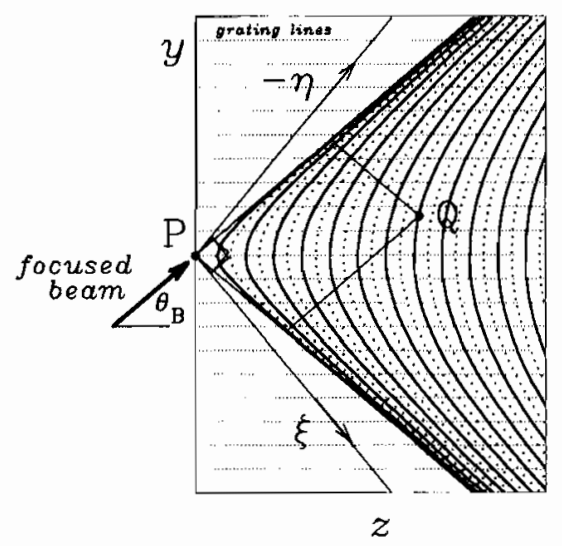

Figure A1. Geometry of Green's function for singly periodic structure in nearly free photon case; the position of the intensity peaks for the Bragg reflected and transmitted fields are given by the zeros and peaks in the Bessel functions (A11).

\section{APPENDIX A3. BESSEL FUNCTION SOLUTIONS}

\section{A3.1 Nearly-Free Photons: Green's Function Solution²7,31}

When (1) the index modulation is very weak, i.e. $\epsilon_{m}=n_{1}^{2}-n_{2}^{2} \ll \epsilon_{\mathrm{av}}$, (2) the Bragg angle $\theta_{\mathrm{B}}=\arcsin \left(K / 2 k n_{\mathrm{av}}\right)$ lies in the mid-range between 0 and $\pi$, (3) a beam B is focused to a small point on the boundary at $P$ (see Figure A1) and (4) its plane wave spectrum is centred at the Bragg angle, then the transmitted and Bragg reflected field intensities at an arbitrary point $Q$ are given by:

$$
\begin{aligned}
& T_{Q P}=\mathrm{J}_{0}^{2}\left(\frac{2 \kappa}{\sin \theta_{B}} \sqrt{\eta_{Q} \xi_{Q}}\right) \\
& R_{Q P}=\frac{\eta_{Q}}{\xi_{Q}} \mathrm{~J}_{1}^{2}\left(\frac{2 \kappa}{\sin \theta_{B}} \sqrt{\eta_{Q} \xi_{Q}}\right)
\end{aligned}
$$

where the coordinates $\xi$ and $\eta$ are defined by: 


$$
\left(\begin{array}{l}
\xi \\
\eta
\end{array}\right)=\left(\begin{array}{cc}
\sin \theta_{B} & -\cos \theta_{B} \\
-\sin \theta_{B} & -\cos \theta_{B}
\end{array}\right)\left(\begin{array}{l}
z \\
y
\end{array}\right)
$$

where $\kappa=\epsilon_{\mathrm{m}} k / 4 n_{\mathrm{av}}$ is known as the coupling constant. This solution arises when the incident beam $B$ has an angular spectrum large enough to excite Bloch waves over each entire stop-band branch, while the stop-band itself is small, i.e., the beam $B$ has an angular spectrum small enough for it not to diffract appreciably in the absence of a grating $(\kappa \rightarrow$ 0 ). The minimum feature size needed to excite this function is of order $\Delta y=2 \pi / k \sin \theta_{\mathrm{B}}$. Note that the form of the solution does not depend on the boundary slant angle, provided it does not cut into the triangular region bounded by the Bragg angles. Because they are obtained by integration over the entire stop-band, these functions are akin (though not identical) to Wannier functions in electronic band theory ${ }^{23,25}$. They are also Green's functions, and can be used to build up more complicated solutions by linear superposition; e.g., non-planar boundary shapes and non-uniform incident beams can be treated. The solution they represent is in the "nearly free photon" approximation.

\section{A3.2 Tight Binding}

Excitation of a single waveguide in an array of parallel coupled channel waveguides (or a single tightly bound high index layer in a multi-layer stack - see section 6.2 ) results in lateral spreading out of power by resonant tunnelling. After propagating a distance $z$, the power in the $n$th channel (the entrant channel being $n=0$, see Figure A2) is given by:

$$
P_{n}(z)=\mathrm{J}_{n}^{2}\left(2 \kappa_{c} z\right)
$$

where the inter-guide coupling constant $\kappa_{c}$ is a function of the waveguide parameters ${ }^{52}$. The tight-binding approximation upon which this solution is based is common in waveguide optics, where it is formulated in terms of coupled mode theory. Again, because the structure is excited at one lattice point, the field at entry to the structure is akin to an electronic Wannier function.

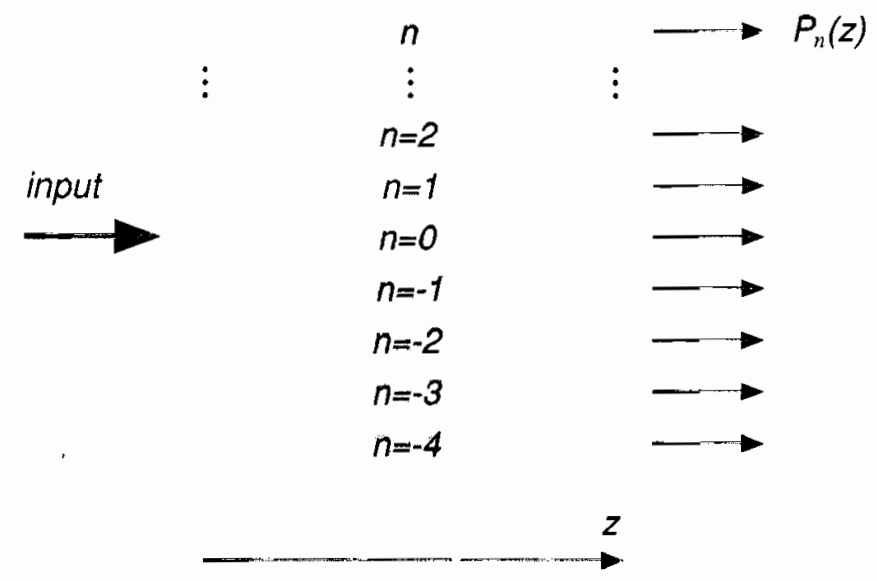

Figure A2. Geometry of the coupled waveguide array for the tight-binding Bessel function solution in (A13). 